\title{
Higher Order Mortar Finite Element Methods in 3D with Dual Lagrange Multiplier Bases ${ }^{\star}$
}

\author{
B. P. Lamichhane ${ }^{1}$, R. P. Stevenson ${ }^{2}$, B. I. Wohlmuth ${ }^{1}$ \\ 1 Institute of Applied Analysis and Numerical Simulation, University of Stuttgart, \\ Germany, e-mail: \{lamichhane, wohlmuth\}@mathematik.uni-stuttgart.de \\ 2 Department of Mathematics, Utrecht University, The Netherlands, \\ e-mail: stevenson@math.uu.nl
}

The date of receipt and acceptance will be inserted by the editor

Summary Mortar methods with dual Lagrange multiplier bases provide a flexible, efficient and optimal way to couple different discretization schemes or nonmatching triangulations. Here, we generalize the concept of dual Lagrange multiplier bases by relaxing the condition that the trace space of the approximation space at the slave side with zero boundary condition on the interface and the Lagrange multiplier space have the same dimension. We provide a new theoretical framework within this relaxed setting, which opens a new and simpler way to construct dual Lagrange multiplier bases for higher order finite element spaces. As examples, we consider quadratic and cubic tetrahedral elements and quadratic serendipity hexahedral elements. Numerical results illustrate the performance of our approach.

Key words. Mortar finite elements, Lagrange multipliers, biorthogonal bases, nonmatching triangulations.

AMS subject classification. 35N55, 65N30.

\section{Introduction}

Over the recent past years, mortar methods with dual Lagrange multiplier bases have become an active area of research, see, e.g., [27,

\footnotetext{
* This work was supported in part by the Deutsche Forschungsgemeinschaft, SFB 404, C12, the Netherlands Organization for Scientific Research and by the European Community's Human Potential Programme under contract HPRN-CT2002-00286.
} 
$30,17,28,18,19]$. Originally introduced to couple spectral and finite element approximations [5-7], mortar methods have a wide range of applications. Being a powerful tool to couple different discretization schemes or nonmatching triangulations, these nonconforming techniques provide a more flexible approach than standard conforming approaches. Examples where such situations occur are when, in a parallel environment, mesh generators are used independently on different subdomains, or when different physical models or, within one model, strongly different parameter values naturally lead to the use of different discretization schemes on different subdomains. The central idea of mortar techniques is to replace the strong, pointwise continuity across the interface by a weak one, which requires that the jump of the solution across the interface is orthogonal to a suitable Lagrange multiplier space. Under this relaxed condition, optimal a priori estimates of the discretization error have been demonstrated, cf. $[3,2,7,28,17]$, and various iterative methods have been introduced to solve the arising linear system, see $[9,8,30,14,26]$.

Generally, the mortar approach has the disadvantage that even when the boundary value problem is elliptic, the arising linear system is of saddle point type, usually for which iterative methods are known to be less efficient than for symmetric positive definite systems. However, when working with dual Lagrange multiplier bases, the degrees of freedom associated to the multiplier can be locally eliminated leading to a sparse, positive definite system, on which, for example, efficient multigrid methods can be applied, see [30,28]. For the lowest order finite elements in 3D, dual Lagrange multiplier bases were constructed in $[17,28]$. However, the construction of such bases for higher order elements is more complicated, see $[18,19,22]$. In the present paper, we develop a general framework facilitating the construction of Lagrange multiplier bases, in particular for higher order elements. In a first step, we remove the condition that the trace space of the approximation space at the slave side with zero boundary condition on the interface and the Lagrange multiplier space have the same dimension, with which the introduction of additional degrees of freedom at the multiplier side only to satisfy this condition on the dimensions is avoided. As a result, we can work with a subspace of the trace space for which it is easier to construct a dual Lagrange multiplier basis. In a second step, we describe a general procedure for the construction of multiplier basis functions near the interface boundaries, where one has to deal with the complication that homogeneous boundary conditions are incorporated in the trace space of the approximation space at the slave side, but not in the Lagrange 
multiplier space. Although the theory presented in this paper applies to general space dimensions, our concrete realizations of dual Lagrange multiplier bases will take place in the three dimensional case.

This paper is organized as follows: In the rest of this section, we describe the problem setting, and fix some notations related to the mortar method. In Section 2, we prove some optimal a priori estimates without assuming that the trace space with zero boundary condition on the interface from the slave side has the same dimension as the multiplier space. As in $[17,12]$, we allow locally refined finite element partitions and geometrically nonconforming subdivisions into subdomains. Furthermore, as in $[19,12]$, we do not require that the partitions match on the boundaries of the interfaces. For the case of having quasi-uniform meshes on each subdomain, we will prove an error estimate showing that the possibly disadvantageous effect of having at the slave side a finer mesh than at the master side is much milder than that is indicated by existing error estimates, e.g., in $[28,19]$. A general framework for the construction of dual multiplier bases, in particular for the modifications needed along the interface boundaries, is given in Section 3. Examples of dual multiplier bases are given for quadratic and cubic tetrahedral elements, and for quadratic serendipity hexahedral elements. Finally, in Section 4, we present some numerical results concerning the discretization errors in the $L^{2}$-norm, the piecewise $H^{1}$-norm, and in a weighted $L^{2}$-norm for the Lagrange multiplier.

We consider the following elliptic second order boundary value problem in variational form: Find $u \in H_{0}^{1}(\Omega)$ such that

$$
\int_{\Omega} a \nabla u \cdot \nabla v+c u v d x=f(v), \quad v \in H_{0}^{1}(\Omega),
$$

where $\Omega \subset \mathbb{R}^{d}$ is a bounded polytope, $a, c \in L^{\infty}(\Omega), a>a_{0}>0$ and $c \geq 0$. The domain $\Omega$ is decomposed into $K$ non-overlapping polytopes $\Omega_{k}$, i.e.,

$$
\bar{\Omega}=\bigcup_{k=1}^{K} \bar{\Omega}_{k} \quad \text { with } \quad \Omega_{k} \cap \Omega_{\ell}=\emptyset \quad \text { for } \quad k \neq \ell .
$$

Defining, for each $1 \leq k \leq K$,

$$
H_{*}^{1}\left(\Omega_{k}\right):=\left\{v \in H^{1}\left(\Omega_{k}\right), v_{\mid \partial \Omega \cap \partial \Omega_{k}}=0\right\},
$$

we assume that $f \in \prod_{k=1}^{K}\left(H_{*}^{1}\left(\Omega_{k}\right)\right)^{\prime}$. We assume that $a$ and $c$ are piecewise smooth with respect to the subdivision into subdomains, but 
allow that they have jumps over the interfaces. However, in this paper, no attempt is made to obtain results that hold uniformly in the sizes of such jumps, cf. [16].

For any $\{k, \ell\}$ with $1 \leq k \neq \ell \leq K$, for which the closures of $\Omega_{k}$ and $\Omega_{\ell}$ have a $(d-1)$-dimensional intersection, we set $\bar{\gamma}_{i}=\bar{\Omega}_{k} \cap \bar{\Omega}_{\ell}$, where $1 \leq i \leq N$ is a number uniquely associated to this $\{k, \ell\}$, and thus with $N$ being the total number of sets $\{k, \ell\}$ having this property. For each $1 \leq i \leq N$, one of the subdomains $\Omega_{k}$ and $\Omega_{\ell}$ on both sides of the interface is referred to be at the slave or non-mortar side, and its index is denoted as $s(i)$, where the other is referred to be at the master or mortar side, with its index denoted as $m(i)$.

For each $\delta$ from some index set $\mathcal{I}$, and each $1 \leq k \leq K$, we assume a finite element space $X_{k}^{\delta} \subset H_{*}^{1}\left(\Omega_{k}\right)$ with respect to a partition of $\Omega_{k}$ into "elements", e.g., $d$-simplices or $d$-rectangles. As examples, one may think of $X_{k}^{\delta}$ as being a finite element space with respect to some uniform refinement of an initial partition of $\Omega_{k}$, or as being such a space with respect to some locally refined partition generated by an adaptive procedure. In the latter case, generally $\prod_{k=1}^{K} X_{k}^{\delta}$ cannot be parametrized by the maximum or minimum diameter of any underlying element, since these values may not uniquely define a space from the whole family of spaces that has been created. Therefore, we use some general, but for the rest meaningless parameter $\delta \in \mathcal{I}$. Its only function is to link conditions with statements, in the sense that if some conditions are valid uniformly in $\delta \in \mathcal{I}$, i.e., uniformly over some general family of spaces, then some statements will be valid uniformly in $\delta \in \mathcal{I}$.

We assume that for any $1 \leq i \leq N$ and $\delta \in \mathcal{I}$,

$$
\left\{\begin{array}{l}
\gamma_{i} \text { is the union of complete }(d-1) \text {-dimensional faces of } \\
\text { elements underlying } X_{s(i)}^{\delta} .
\end{array}\right.
$$

Note that (A.1) does allow the partition into subdomains to be geometrically nonconforming; $\gamma_{i}$ needs not to be a full face of both $\Omega_{m(i)}$ and $\Omega_{s(i)}$, or even not to be a full face of either $\Omega_{m(i)}$ or $\Omega_{s(i)}$, see Figure 1.1. In case the decomposition into subdomains is geometrically conforming, (A.1) is automatically satisfied.

For $1 \leq i \leq N$ and $\delta \in \mathcal{I}$, we define the trace space

$$
W_{0, i}^{\delta}=\left\{\left.v^{\delta}\right|_{\gamma_{i}}: v^{\delta} \in X_{s(i)}^{\delta},\left.v^{\delta}\right|_{\partial \Omega_{s(i)} \backslash \gamma_{i}}=0\right\} .
$$

As a consequence of $X_{s(i)}^{\delta} \subset H_{*}^{1}\left(\Omega_{s(i)}\right)$ being a finite element space, we can assume that $W_{0, i}^{\delta} \subset H_{0}^{1}\left(\gamma_{i}\right)$. We will refer to the partition of $\gamma_{i}$ into the faces of elements as referred to in (A.1) as the finite element 


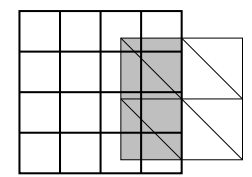

Fig. 1.1: An interface that is not a full face of either $\Omega_{m(i)}$ or $\Omega_{s(i)}$; it satisfies (A.1) when the square partitioned into triangles is a face of $\Omega_{s(i)}$, but not when this is a face of $\Omega_{m(i)}$.

partition underlying $W_{0, i}^{\delta}$. For enforcing a weak coupling of the finite element functions over the interfaces, for each $1 \leq i \leq N$ and $\delta \in \mathcal{I}$ we introduce a Lagrange multiplier space $M_{i}^{\delta} \subset L^{2}\left(\gamma_{i}\right)$. Thinking of $M_{i}^{\delta}$ as being a finite element space, the partition underlying $M_{i}^{\delta}$ will be equal to that of $W_{0, i}^{\delta}$.

Setting the product spaces $X^{\delta}:=\prod_{k=1}^{K} X_{k}^{\delta}, M^{\delta}:=\prod_{i=1}^{N} M_{i}^{\delta}$, and defining the bilinear forms $a(v, w)=\sum_{k=1}^{K} \int_{\Omega_{k}} a \nabla v \cdot \nabla w+c v w d x$ and $b(v, \mu)=\sum_{i=1}^{N} \int_{\gamma_{i}}[v]_{\gamma_{i}} \mu d \sigma$, where $[v]_{\gamma_{i}}$ denotes the jump of $v$ on $\gamma_{i}$ from the master to the slave side, the mortar finite element discretization now consists in finding $\left(u^{\delta}, \lambda^{\delta}\right) \in X^{\delta} \times M^{\delta}$ such that

$$
\begin{aligned}
a\left(u^{\delta}, v^{\delta}\right)+b\left(v^{\delta}, \lambda^{\delta}\right) & =f\left(v^{\delta}\right), & & v^{\delta} \in X^{\delta}, \\
b\left(u^{\delta}, \mu^{\delta}\right) & =0, & & \mu^{\delta} \in M^{\delta},
\end{aligned}
$$

or, considering the first component only, in finding

$$
u^{\delta} \in V^{\delta}:=\left\{v^{\delta} \in X^{\delta}: b\left(v^{\delta}, \mu^{\delta}\right)=0, \mu^{\delta} \in M^{\delta}\right\}
$$

such that

$$
a\left(u^{\delta}, v^{\delta}\right)=f\left(v^{\delta}\right), \quad v^{\delta} \in V^{\delta} .
$$

Assuming that for any $\delta \in \mathcal{I}$ and $1 \leq i \leq N$, the constant function

$$
1 \in M_{i}^{\delta} \text {, }
$$

and that

$$
\beta_{i}^{\delta}:=\inf _{\mu_{i}^{\delta} \in M_{i}^{\delta} \backslash\{0\}} \sup _{w_{i}^{\delta} \in W_{0, i}^{\delta} \backslash\{0\}} \frac{\left\langle\mu_{i}^{\delta}, w_{i}^{\delta}\right\rangle_{L^{2}\left(\gamma_{i}\right)}}{\left\|\mu_{i}^{\delta}\right\|_{L^{2}\left(\gamma_{i}\right)}\left\|w_{i}^{\delta}\right\|_{L^{2}\left(\gamma_{i}\right)}}>0,
$$

the problem (1.2) and thus (1.3) are known to have a unique solution, cf. $[7,10]$.

In this paper, in order to avoid the repeated use of generic but unspecified constants, by $C \lesssim D$ we mean that $C$ can be bounded by a multiple of $D$, independently of parameters which $C$ and $D$ may depend on, where we, in particular, think of $\delta$ as well as the number of subdomains $K$. Obviously, $C \gtrsim D$ is defined as $D \lesssim C$, and $C \approx D$ as $C \lesssim D$ and $C \gtrsim D$. 


\section{A priori estimates}

We start with establishing suitable mortar projectors under the condition that $\beta_{i}^{\delta} \gtrsim 1$ in (1.4). Note that (1.4) can only be valid when $\operatorname{dim} W_{0, i}^{\delta} \geq \operatorname{dim} M_{i}^{\delta}$, but, on the other hand, that it does not require that $\operatorname{dim} W_{0, i}^{\delta}=\operatorname{dim} M_{i}^{\delta}$, which is usually imposed in the literature.

Lemma 2.1 Let $M, W$ be closed subspaces of some Hilbert space $H$, with scalar product $\langle\cdot, \cdot\rangle$ and associated norm $\|\cdot\|$. Then

$$
\beta:=\inf _{\mu \in M \backslash\{0\}} \sup _{w \in W \backslash\{0\}} \frac{\langle\mu, w\rangle}{\|\mu\|\|w\|}>0,
$$

if and only if there exists a bounded projector $\Pi$ from $H$ onto $W$ for which

$$
\langle\Pi v, \mu\rangle=\langle v, \mu\rangle \quad v \in H, \mu \in M .
$$

Moreover, $\beta^{-1} \leq\|\Pi\| \leq 1+2 \beta^{-1}$.

Proof Assuming the existence of the bounded projector $\Pi$, for any $\mu \in M \backslash\{0\}$ we have

$$
\sup _{w \in W \backslash\{0\}} \frac{\langle\mu, w\rangle}{\|\mu\|\|w\|} \geq \frac{\langle\mu, \Pi \mu\rangle}{\|\mu\|\|\Pi \mu\|} \geq\|\Pi\|^{-1} .
$$

On the other hand, when $\beta>0$, given $v \in H$, define $(w, \lambda) \in W \times M$ as the solution of

$$
\begin{array}{ll}
\langle w, z\rangle+\langle\lambda, z\rangle & =\langle v, z\rangle, \quad z \in W, \\
\langle w, \mu\rangle & =\langle v, \mu\rangle, \quad \mu \in M .
\end{array}
$$

It is well known (see e.g. [10, $\S$ II.1, Proposition 1.3]) that this saddle point problem has a unique solution with $\|w\| \leq\left(1+2 \beta^{-1}\right)\|v\|$. When $v \in W$, the second equation shows that $v-w \perp M$. Substituting $z=v-w$, we conclude that $w=v$, so that $\Pi: v \mapsto w$ is a projector.

We assume that for $1 \leq i \leq N, \delta \in \mathcal{I}$ there exist $0<\underline{h}_{i}^{\delta} \in L^{\infty}\left(\gamma_{i}\right)$ such that

$$
\left\|w_{i}^{\delta}\right\|_{H^{1}\left(\gamma_{i}\right)} \lesssim\left\|\left(\underline{h}_{i}^{\delta}\right)^{-1} w_{i}^{\delta}\right\|_{L^{2}\left(\gamma_{i}\right)}, \quad w_{i}^{\delta} \in W_{0, i}^{\delta}
$$

and

$\inf _{w_{i}^{\delta} \in W_{0, i}^{\delta}}\left\|\left(\underline{h}_{i}^{\delta}\right)^{-1}\left(w-w_{i}^{\delta}\right)\right\|_{L^{2}\left(\gamma_{i}\right)}+\left\|w_{i}^{\delta}\right\|_{H^{1}\left(\gamma_{i}\right)} \lesssim\|w\|_{H^{1}\left(\gamma_{i}\right)}, w \in H_{0}^{1}\left(\gamma_{i}\right)$.

If the finite element partition underlying $W_{0, i}^{\delta}$ is shape regular and quasi-uniform, both uniformly in $\delta$, then with $\underline{h}_{i}^{\delta}$ being the constant 
function defined as the maximum of the diameters of the elements, (A.3) is the well-known inverse inequality, and (A.4) is guaranteed by the minimal requirement that $W_{0, i}^{\delta}$ contains all continuous functions that vanish at $\partial \gamma_{i}$ and that are piecewise linear with respect to the partition. Yet, for partitions that, instead of quasi-uniform, are only locally quasi-uniform, meaning that any two elements that have non-empty intersection have uniformly comparable diameters, (A.3) and (A.4) are also valid, now with $\underline{h}_{i}^{\delta}$ being defined as the piecewise constant function equal to the diameter of the underlying element. See, e.g., $[11,23]$.

We assume the existence of projectors $\Pi_{i}^{\delta}: L^{2}\left(\gamma_{i}\right) \rightarrow L^{2}\left(\gamma_{i}\right)$ onto $W_{0, i}^{\delta}$ with

$$
\left\langle\Pi_{i}^{\delta} v, \mu_{i}^{\delta}\right\rangle_{L^{2}\left(\gamma_{i}\right)}=\left\langle v, \mu_{i}^{\delta}\right\rangle_{L^{2}\left(\gamma_{i}\right)} \quad v \in L^{2}\left(\gamma_{i}\right), \mu_{i}^{\delta} \in M_{i}^{\delta} .
$$

which are called mortar projectors, that satisfy

$$
\left\|\Pi_{i}^{\delta}\right\|_{L_{2}\left(\gamma_{i}\right) \rightarrow L_{2}\left(\gamma_{i}\right)} \lesssim 1
$$

As follows from Lemma 2.1, such projectors exist if and only if $\beta_{i} \gtrsim 1$. The mortar projectors are uniquely determined by (2.1) only when $\operatorname{dim} W_{i, 0}^{\delta}=\operatorname{dim} M_{i}^{\delta}$. For the case that not all $\underline{h}_{i}^{\delta}$ are constant functions, we make the additional assumption that they can be selected such that also

$$
\left\|\left(\underline{h}_{i}^{\delta}\right)^{-1} \Pi_{i}^{\delta} w\right\|_{L^{2}\left(\gamma_{i}\right)} \lesssim\left\|\left(\underline{h}_{i}^{\delta}\right)^{-1} w\right\|_{L^{2}\left(\gamma_{i}\right)}, \quad w \in L^{2}\left(\gamma_{i}\right) .
$$

Again assuming that the partition underlying $W_{i, 0}^{\delta}$ is shape regular and locally quasi-uniform, (A.6) is valid when the $\Pi_{i}^{\delta}$ are local in the sense that $\Pi_{i}^{\delta} v$ vanishes on an element $T$ whenever $v$ vanishes on all elements that have distance to $T$ less than some absolute multiple of $\operatorname{diam}(T)$.

Lemma 2.2 From (A.3), (A.6) and (A.4), it follows that $\Pi_{i}^{\delta}: H_{0}^{1}\left(\gamma_{i}\right) \rightarrow$ $H_{0}^{1}\left(\gamma_{i}\right)$ is bounded, uniformly in $\delta$ and $i$.

Proof Since $\Pi_{i}^{\delta}$ is a projector onto $W_{0, i}^{\delta}$, by (A.3), (A.6) and (A.4) for $w \in H_{0}^{1}\left(\gamma_{i}\right)$ we have

$$
\begin{aligned}
\left\|\Pi_{i}^{\delta} w\right\|_{H^{1}\left(\gamma_{i}\right)} & \lesssim \inf _{w_{i}^{\delta} \in W_{0, i}^{\delta}}\left\|\Pi_{i}^{\delta}\left(w-w_{i}^{\delta}\right)\right\|_{H^{1}\left(\gamma_{i}\right)}+\left\|w_{i}^{\delta}\right\|_{H^{1}\left(\gamma_{i}\right)} \\
& \lesssim \inf _{w_{i}^{\delta} \in W_{0, i}^{\delta}}\left\|\left(\underline{\underline{h}}_{i}^{\delta}\right)^{-1} \Pi_{i}^{\delta}\left(w-w_{i}^{\delta}\right)\right\|_{L^{2}\left(\gamma_{i}\right)}+\left\|w_{i}^{\delta}\right\|_{H^{1}\left(\gamma_{i}\right)} \\
& \lesssim \inf _{w_{i}^{\delta} \in W_{0, i}^{\delta}}\left\|\left(\underline{h}_{i}^{\delta}\right)^{-1}\left(w-w_{i}^{\delta}\right)\right\|_{L^{2}\left(\gamma_{i}\right)}+\left\|w_{i}^{\delta}\right\|_{H^{1}\left(\gamma_{i}\right)} \\
& \lesssim\|w\|_{H^{1}\left(\gamma_{i}\right)},
\end{aligned}
$$


which shows the second statement.

Finally, for completeness, we mention the following technical assumption:

$$
\left\{\begin{array}{l}
\text { There exist } J_{k}^{\delta}: H^{1}\left(\Omega_{k}\right) \rightarrow X_{k}^{\delta} \text { with }\left\|J_{k}^{\delta}\right\|_{H^{1}\left(\Omega_{k}\right) \rightarrow H^{1}\left(\Omega_{k}\right)} \lesssim 1 \\
\text { such that whenever }\left.v\right|_{\partial \Omega_{k}}=\left.v_{k}^{\delta}\right|_{\partial \Omega_{k}} \text { for some } v_{k}^{\delta} \in X_{k}^{\delta}, \\
\text { then }\left.\left(J_{k}^{\delta} v\right)\right|_{\partial \Omega_{k}}=\left.v_{k}^{\delta}\right|_{\partial \Omega_{k}} .
\end{array}\right.
$$

For any given $X_{k}^{\delta}$, the existence of such $J_{k}^{\delta}$ can be demonstrated as in $[23]$.

Under the assumptions we made, and using Lemma 2.2, the error estimates proven in [19] easily generalize to the present more general setting. See $[2,17,12]$ for preceding or related work.

Theorem 2.3 For $u$ and $\left(u^{\delta}, \lambda^{\delta}\right)$ being the solutions of (1.1) and (1.2), respectively, we have

$$
\begin{aligned}
& \sum_{k=1}^{K}\left\|u-u^{\delta}\right\|_{H^{1}\left(\Omega_{k}\right)}^{2} \lesssim \inf _{v^{\delta} \in V^{\delta}} \sum_{k=1}^{K}\left\|u-v^{\delta}\right\|_{H^{1}\left(\Omega_{k}\right)}^{2} \\
& \sup _{v^{\delta} \in V^{\delta} \backslash\{0\}} \inf _{\mu^{\delta} \in M^{\delta}} b\left(v^{\delta}, a \frac{\partial u}{\partial \mathbf{n}_{\gamma_{i}}}-\mu^{\delta}\right)^{2} / \sum_{k=1}^{N}\left\|v^{\delta}\right\|_{H^{1}\left(\Omega_{k}\right)}^{2},
\end{aligned}
$$

where

$$
\begin{aligned}
\inf _{v^{\delta} \in V^{\delta}} & \sum_{k=1}^{K}\left\|u-v^{\delta}\right\|_{H^{1}\left(\Omega_{k}\right)}^{2} \lesssim \inf _{x^{\delta} \in X^{\delta}}\left\{\sum_{k=1}^{K}\left\|u-x^{\delta}\right\|_{H^{1}\left(\Omega_{k}\right)}^{2}+\right. \\
& \left.\sum_{i=1}^{N}\left\|\left.\left(\underline{h}_{i}^{\delta}\right)^{-\frac{1}{2}}\left(u-x^{\delta}\right)\right|_{\Omega_{m(i)}}\right\|_{L^{2}\left(\gamma_{i}\right)}^{2}+\left\|\left.\left(\underline{h}_{i}^{\delta}\right)^{-\frac{1}{2}}\left(u-x^{\delta}\right)\right|_{\Omega_{s(i)}}\right\|_{L^{2}\left(\gamma_{i}\right)}^{2}\right\} .
\end{aligned}
$$

With $\mathbf{n}_{\gamma_{i}}$ being the outward normal on $\gamma_{i}$ from the master side, for the Lagrange multiplier we have

$$
\begin{aligned}
\sum_{i=1}^{N} \| a \frac{\partial u}{\partial \mathbf{n}_{\gamma_{i}}} & -\lambda^{\delta} \|_{\left(H_{00}^{\frac{1}{2}}\left(\gamma_{i}\right)\right)^{\prime}}^{2} \\
& \lesssim \sum_{k=1}^{K}\left\|u-u^{\delta}\right\|_{H^{1}\left(\Omega_{k}\right)}^{2}+\inf _{\mu^{\delta} \in M^{\delta}} \sum_{i=1}^{N}\left\|a \frac{\partial u}{\partial \mathbf{n}_{\gamma_{i}}}-\mu^{\delta}\right\|_{\left(H_{00}\right.}^{\left.\frac{1}{2}\left(\gamma_{i}\right)\right)^{\prime}}
\end{aligned}
$$

Proof As shown in [6], the coercivity $a\left(v^{\delta}, v^{\delta}\right) \gtrsim \sum_{k=1}^{K}\left\|v^{\delta}\right\|_{H^{1}\left(\Omega_{k}\right)}^{2}$ for $v^{\delta} \in V^{\delta}$ is a consequence of (A.2), from which (2.2) follows using Strang's second lemma as in [19, proof of Theorem 2.3]. Because 
of (A.7), any $w_{i}^{\delta} \in W_{0, i}^{\delta}$ has an extension to a $x_{s(i)}^{\delta} \in X_{s(i)}^{\delta}$ with $\left\|x_{s(i)}^{\delta}\right\|_{H^{1}\left(\Omega_{s(i)}\right)} \lesssim\left\|w_{i}^{\delta}\right\|_{H_{00}^{\frac{1}{2}\left(\gamma_{i}\right)}}$ and $\left.x_{s(i)}^{\delta}\right|_{\partial \Omega_{s(i)} \backslash \gamma_{i}}=0$, see, e.g., $[24$, Lemma 5.1]. Now, following [19, proof Lemma 2.2], one derives that

$$
\begin{aligned}
& \inf _{v^{\delta} \in V^{\delta}} \sum_{k=1}^{K}\left\|u-v^{\delta}\right\|_{H^{1}\left(\Omega_{k}\right)}^{2} \lesssim \\
& \inf _{x^{\delta} \in X^{\delta}} \sum_{k=1}^{K}\left\|u-x^{\delta}\right\|_{H^{1}\left(\Omega_{k}\right)}^{2}+\sum_{i=1}^{N}\left\|\Pi_{i}^{\delta}\left[u-x^{\delta}\right]_{\gamma_{i}}\right\|_{H_{00}^{\frac{1}{2}\left(\gamma_{i}\right)}}^{2} .
\end{aligned}
$$

From (A.3) and interpolation (cf. [25, ex. in $\$ 1.15 .3]$ ), we infer that $\left\|w_{i}^{\delta}\right\|_{H_{00}^{\frac{1}{2}\left(\gamma_{i}\right)}} \lesssim\left\|\left(\underline{h}_{i}^{\delta}\right)^{-\frac{1}{2}} w_{i}^{\delta}\right\|_{L^{2}\left(\gamma_{i}\right)}$ for $w_{i}^{\delta} \in W_{0, i}^{\delta}$, and from (A.5), (A.6) and interpolation, we have $\left\|\left(\underline{h}_{i}^{\delta}\right)^{-\frac{1}{2}} \Pi_{i}^{\delta} w\right\|_{L^{2}\left(\gamma_{i}\right)} \lesssim\left\|\left(\underline{h}_{i}^{\delta}\right)^{-\frac{1}{2}} w\right\|_{L^{2}\left(\gamma_{i}\right)}$ for any $w \in L^{2}\left(\gamma_{i}\right)$. Combining both results with (2.5) shows (2.3).

The bound (2.4) for the Lagrange multiplier follows from [2, Lemma 2.7 , where the uniform inf-sup condition from [2, Proposition 2.6] can be shown as in [19, Lemma 2.4] using the above extension operator and the fact that $\Pi_{i}^{\delta}: H_{00}^{\frac{1}{2}}\left(\gamma_{i}\right) \rightarrow H_{00}^{\frac{1}{2}}\left(\gamma_{i}\right)$ is uniformly bounded. This follows by interpolation from (A.5) and Lemma 2.2, using that $\left[H_{0}^{1}\left(\gamma_{i}\right), L^{2}\left(\gamma_{i}\right)\right]_{1 / 2}$ and $H_{00}^{1 / 2}\left(\gamma_{i}\right)$ agree as sets and have equivalent norms.

Corollary 2.4 For $1 \leq k \leq N$ and $\delta \in \mathcal{I}$, let $\frac{3}{2}<t_{k} \notin \mathbb{N}+\frac{1}{2}$ and $0<h_{k}^{\delta} \in L^{\infty}\left(\Omega_{k}\right)$ be such that $\underline{h}_{i}^{\delta}:=\left.h_{s(i)}^{\delta}\right|_{\gamma_{i}} \in L^{\infty}\left(\gamma_{i}\right)$ and, with this $\underline{h}_{i}^{\delta}$, (A.3), (A.4) and (A.6) are valid, and

$$
\begin{aligned}
\inf _{x_{k}^{\delta} \in X_{k}^{\delta}} \| x_{k}- & \left.x_{k}^{\delta}\left\|_{H^{1}\left(\Omega_{k}\right)}+\right\|\left(h_{k}^{\delta}\right)^{-\frac{1}{2}}\left(x_{k}-x_{k}^{\delta}\right)\right|_{\partial \Omega_{k}} \|_{L^{2}\left(\partial \Omega_{k}\right)} \\
& \lesssim\left\|h_{k}^{\delta}\right\|_{L^{\infty}\left(\Omega_{k}\right)}^{t_{k}-1}\left\|x_{k}\right\|_{H^{t_{k}}\left(\Omega_{k}\right)}, \quad x_{k} \in H_{*}^{1}\left(\Omega_{k}\right) \cap H^{t_{k}}\left(\Omega_{k}\right), \\
\inf _{\mu_{i}^{\delta} \in M_{i}^{\delta}} \| \mu_{i}- & \mu_{i}^{\delta}\left\|_{L^{2}\left(\gamma_{i}\right)} \lesssim\right\| \underline{h}_{i}^{\delta}\left\|_{L^{\infty}\left(\gamma_{i}\right)}^{t_{s(i)}-1}\right\| \mu_{i} \|_{H^{t_{s(i)}-1}\left(\gamma_{i}\right)}, \quad \mu_{i} \in H^{t_{s(i)}-1}\left(\gamma_{i}\right) .
\end{aligned}
$$

Assume that the solution $u$ of (1.1) is in $\prod_{k=1}^{K} H^{t_{k}}\left(\Omega_{k}\right)$. Then the solution $\left(u^{\delta}, \lambda^{\delta}\right)$ of (1.2) satisfies

$$
\begin{aligned}
\sum_{k=1}^{K}\left\|u-u^{\delta}\right\|_{H^{1}\left(\Omega_{k}\right)}^{2}+\sum_{i=1}^{N}\left\|a \frac{\partial u}{\partial \mathbf{n}_{\gamma_{i}}}-\lambda^{\delta}\right\|_{\left(H_{00}^{\frac{1}{2}}\left(\gamma_{i}\right)\right)^{\prime}}^{2} \lesssim \\
{\left[1+\max _{1 \leq i \leq N}\left\|h_{m(i)}^{\delta} / h_{s(i)}^{\delta}\right\|_{L^{\infty}\left(\gamma_{i}\right)}\right] \sum_{k=1}^{K}\left\|h_{k}^{\delta}\right\|_{L^{\infty}\left(\Omega_{k}\right)}^{2 t_{k}-2}\|u\|_{H^{t_{k}\left(\Omega_{k}\right)}}^{2} . }
\end{aligned}
$$


Proof Using (2.6), the right-hand side of (2.3) can be estimated on the right-hand side of (2.8). By the trace theorem, the term $\sup _{v^{\delta} \in V^{\delta} \backslash\{0\}} \inf _{\mu^{\delta} \in M^{\delta}} b\left(v^{\delta}, a \frac{\partial u}{\partial \mathbf{n}_{\gamma_{i}}}-\mu^{\delta}\right)^{2} / \sum_{k=1}^{N}\left\|v^{\delta}\right\|_{H^{1}\left(\Omega_{k}\right)}^{2}$ in (2.2) can be bounded on $\inf _{\mu^{\delta} \in M^{\delta}} \sum_{i=1}^{N}\left\|a \frac{\partial u}{\partial \mathbf{n}_{\gamma_{i}}}-\mu^{\delta}\right\|_{\left(H^{\frac{1}{2}}\left(\gamma_{i}\right)\right)^{2}}^{2}$, which is also an upperbound for $\inf _{\mu^{\delta} \in M^{\delta}} \sum_{i=1}^{N}\left\|a \frac{\partial u}{\partial \mathbf{n}_{\gamma_{i}}}-\mu^{\delta}\right\|_{\left(H_{00}^{\frac{1}{2}}\left(\gamma_{i}\right)\right)^{\prime}}^{2}$ from (2.4). Now by using $\inf _{\mu_{i}^{\delta} \in M_{i}^{\delta}}\left\|\mu_{i}-\mu_{i}^{\delta}\right\|_{\left(H^{\frac{1}{2}}\left(\gamma_{i}\right)\right)^{\prime}} \lesssim\left\|h_{s(i)}^{\delta}\right\|_{L^{\infty}\left(\Omega_{s(i)}\right.}^{t_{s(i)}-1}\left\|\mu_{i}\right\|_{H^{t_{s(i)}-\frac{3}{2}}\left(\gamma_{i}\right)}$ for any $\mu_{i} \in H^{t_{s(i)}-\frac{3}{2}}\left(\gamma_{i}\right)$, which is a consequence of (2.7), the proof is completed by another application of the trace theorem.

Assuming that $X_{k}^{\delta}$ contains all continuous, and with respect to a shape regular and locally quasi-uniform finite element partition, piecewise polynomials of degree

$$
r_{k}-1 \geq\left\lceil t_{k}\right\rceil-1
$$

that vanish on $\partial \Omega_{k} \cap \partial \Omega$, the estimate (2.6), with $h_{k}^{\delta}$ being the piecewise constant function equal to the diameter of the underlying element, is easily verified using a nodal, or, when $\left\lfloor t_{k}\right\rfloor \leq \frac{d}{2}$, a modified Clément interpolator as introduced in [23]. Similarly, (2.7) is satisfied when $M_{i}^{\delta}$ contains all piecewise, or continuous piecewise polynomials of degree $r_{s(i)}-2$ with respect to the underlying partition inherited from the slave side. Actually, our spaces $M_{i}^{\delta}$ will not completely satisfy these assumptions due to modifications near $\partial \gamma_{i}$. However, as we will see, these modifications will not affect (2.7).

Remark 2.5 Estimate (2.8) requires that $u \in \prod_{k=1}^{K} H^{t_{k}}\left(\Omega_{k}\right)$ with $\min _{k} t_{k}>\frac{3}{2}$, which in case of a discontinuous coefficient $a$ is not necessarily valid. Since the coercivity as mentioned in the proof of Theorem (2.3) shows that $\sum_{k}\left\|u^{\delta}\right\|_{H^{1}\left(\Omega_{k}\right)}^{2} \lesssim a\left(u^{\delta}, u^{\delta}\right) \lesssim\left(\sum_{k}\left\|u^{\delta}\right\|_{H^{1}\left(\Omega_{k}\right)}^{2}\right)^{\frac{1}{2}}$ $\times\left(\sum_{k}\|f\|_{\left(H_{*}^{1}\left(\Omega_{k}\right)\right)^{\prime}}^{\prime}\right)^{\frac{1}{2}}$, for the primal variable an error estimate under weaker regularity assumptions might possibly be derived using an interpolation argument as applied in [4].

The best rate promised by Corollary 2.4 requires that the solution $u \in \prod_{k=1}^{K} H^{r_{k}}\left(\Omega_{k}\right)$, which, in case $\min _{k} r_{k}>2$, even for a convex polygon $\Omega$ cannot be guaranteed by imposing some smoothness conditions on the right-hand side $f$. For less smooth $u$, using suitably locally refined partitions, better results may be expected than those reflected by Corollary 2.4, which, due to the $L^{\infty}$-norms of the $h_{k}^{\delta}$, shows no benefits of local refinements. From the theory of nonlinear approximation, see, e.g. [13], in combination with the bounds 
from Theorem 2.3, we infer that we may hope for the same asymptotic behaviour of the error as function of the number of degrees of freedom under the relaxed assumption that $u$ is the product space $\prod_{k=1}^{K} W_{p}^{r_{k}}\left(\Omega_{k}\right)$, for certain $p<2$, or, more precisely, that it is in such a product space with the Sobolev spaces $W_{p}^{r_{k}}\left(\Omega_{k}\right)$ replaced by certain Besov spaces $B_{p, q}^{r_{k}}\left(\Omega_{k}\right)$. A treatment of this topic is beyond the scope of this paper.

If the finite element partitions from slave and master side coincide at the interface boundaries $\partial \gamma_{i}$ (and there underly the same finite element space), and $t_{k}=t$ for all $1 \leq k \leq K$, then starting from (2.5), and by using Lemma 2.2, (2.8) can be proven without the penalty factor $1+\max _{1 \leq i \leq N}\left\|h_{m(i)}^{\delta} / h_{s(i)}^{\delta}\right\|_{L^{\infty}\left(\gamma_{i}\right)}$. This has been shown in [17] for $t=2$ and $d \leq 3$ by selecting $x^{\delta}$ as the nodal interpolant of $u$, but it extends to arbitrary $d$ and $t>\frac{3}{2}$ by taking $x^{\delta}$ as a quasi-interpolant of $u$ that on $\partial \gamma_{i}$ only depends on $\left.u\right|_{\partial \gamma_{i}} \in L^{1}\left(\partial \gamma_{i}\right)$ (cf. [23]), and by using that for each fixed $\varepsilon>0, H_{00}^{\frac{1}{2}}\left(\gamma_{i}\right)$ and the interpolation space $\left[H_{0}^{\frac{1}{2}+\varepsilon}\left(\gamma_{i}\right), H^{\frac{1}{2}-\varepsilon}\left(\gamma_{i}\right)\right]_{1 / 2}$ agree as sets and have equivalent norms. This "matching condition" on $\partial \gamma_{i}$ is readily met in the two-dimensional case, where the interface boundaries consist of isolated points, but it is an inconvenient restriction in higher dimensions.

In the remainder of this section, assuming quasi-uniform meshes on each subdomain, but without imposing the matching condition, we will show that the penalty factor $1+\max _{1 \leq i \leq N}\left\|h_{m(i)}^{\delta} / h_{s(i)}^{\delta}\right\|_{L^{\infty}\left(\gamma_{i}\right)}$ is much too pessimistic. In Theorem 2.7, we will see that only the log of the quotient of the mesh sizes at master and slave sides, in case this quotient is larger than 1, may enter the upper bound of the error. Although, when having quasi-uniform meshes on each subdomain, for each interface the slave and master sides can always be selected such that the mesh size at the master side is not larger than that at the slave side, it shows that a different choice of master and slave sides can hardly do any harm. Moreover, it indicates that also for locally refined meshes, where uniform boundedness of $\left\|h_{m(i)}^{\delta} / h_{s(i)}^{\delta}\right\|_{L^{\infty}\left(\gamma_{i}\right)}$ cannot always be guaranteed, the penalty factor from Corollary 2.4 is likely too pessimistic, in any case when locally the mesh sizes along the interfaces do not vary too strongly. A generalization of our result to locally refined meshes might be possible by using sufficiently smooth mesh indicator functions $h_{k}^{\delta}$ as used in [12]. However, the estimation of the unavoidable fractional Sobolev norms of functions that contain as factors powers of the non-constant functions $h_{k}^{\delta}$ will require quite some technicalities. 
It is well-known that the interpolation space $\left[H_{0}^{1}\left(\gamma_{i}\right), L^{2}\left(\gamma_{i}\right)\right]_{\theta}$ is equal to $H_{0}^{1-\theta}\left(\gamma_{i}\right)$ for $\theta \in\left[0, \frac{1}{2}\right)$, and that it is equal to $H^{1-\theta}\left(\gamma_{i}\right)$ for $\theta \in\left(\frac{1}{2}, 1\right]$, meaning that corresponding spaces are equal as sets and have equivalent norms. The space $\left[H_{0}^{1}\left(\gamma_{i}\right), L^{2}\left(\gamma_{i}\right)\right]_{1 / 2}$ is strictly contained $H_{0}^{1 / 2}\left(\gamma_{i}\right)$ with a strictly finer topology. In the following we will consider these interpolation spaces for $\theta \downarrow \frac{1}{2}$. We cannot expect that the aforementioned norm equivalences hold uniformly in $\theta \in\left(\frac{1}{2}, 1\right]$ which motivates our following lemma.

Lemma 2.6 For $1 \leq i \leq N$ and $\theta \in[0,1] \backslash\left\{\frac{1}{2}\right\}$, we have

$$
\sup _{v \in\left[H_{0}^{1}\left(\gamma_{i}\right), L^{2}\left(\gamma_{i}\right)\right]_{\theta} \backslash\{0\}} \frac{\|v\|_{\left[H_{0}^{1}\left(\gamma_{i}\right), L^{2}\left(\gamma_{i}\right)\right]_{\theta}}}{\|v\|_{H^{1-\theta}\left(\gamma_{i}\right)}} \lesssim\left|\frac{1}{2}-\theta\right|^{-1} .
$$

Proof We may think of $\gamma_{i}$ as being a polytope in $\mathbb{R}^{d-1}$. For any function $v$ on $\gamma_{i}$, let $\tilde{v}$ denotes its extension on $\mathbb{R}^{d-1}$ with zero. As follows from [20, Lemma 11.3], we have $\|v\|_{\left[H_{0}^{1}\left(\gamma_{i}\right), L^{2}\left(\gamma_{i}\right)\right]_{\theta}} \lesssim\|\tilde{v}\|_{H^{1-\theta}\left(\mathbb{R}^{d-1}\right)}$ uniformly in $u \in\left[H_{0}^{1}\left(\gamma_{i}\right), L^{2}\left(\gamma_{i}\right)\right]_{\theta}$ and $\theta \in[0,1]$. From [15, Lemma 1.3.2.6 and Theorem 1.4.4.4] it follows that for any $\theta \in[0,1] \backslash\left\{\frac{1}{2}\right\}$, there exists a constant $C_{\theta}>0$ such that $\|\tilde{v}\|_{H^{1-\theta}\left(\mathbb{R}^{d-1}\right)} \leq C_{\theta}\|v\|_{H^{1-\theta}\left(\gamma_{i}\right)}$ for all $v \in\left[H_{0}^{1}\left(\gamma_{i}\right), L^{2}\left(\gamma_{i}\right)\right]_{\theta}$. Inspection of the proof of this Theorem 1.4.4.4 reveals that $C_{\theta}$ is a result of the application of Hardy's inequality, and that it can be bounded by some absolute multiple of $\left|\frac{1}{2}-\theta\right|^{-1}$.

Theorem 2.7 For $1 \leq k \leq N$ and $\delta \in \mathcal{I}$, let $\frac{3}{2}<t_{k} \notin \mathbb{N}+\frac{1}{2}$ and $h_{k}>0$ be a constant such that, with $\underline{h}_{i}^{\delta}:=\left.h_{s(i)}^{\delta}\right|_{\gamma_{i}}$, (A.3), (A.4) and (A.6) are valid, and

$$
\begin{aligned}
& \inf _{x_{k}^{\delta} \in X_{k}^{\delta}}\left\|x_{k}-x_{k}^{\delta}\right\|_{H^{1}\left(\Omega_{k}\right)} \\
& \quad \lesssim\left(h_{k}^{\delta}\right)^{t_{k}-1}\left\|x_{k}\right\|_{H^{t_{k}\left(\Omega_{k}\right)}, \quad x_{k} \in H_{*}^{1}\left(\Omega_{k}\right) \cap H^{t_{k}}\left(\Omega_{k}\right),} \\
& \inf _{\mu_{i}^{\delta} \in M_{i}^{\delta}}\left\|\mu_{i}-\mu_{i}^{\delta}\right\|_{L^{2}\left(\gamma_{i}\right)} \lesssim\left(\underline{h}_{i}^{\delta}\right)^{t_{s(i)}-1}\left\|\mu_{i}\right\|_{H^{t_{s(i)}-1}\left(\gamma_{i}\right)}, \quad \mu_{i} \in H^{t_{s(i)}-1}\left(\gamma_{i}\right) .
\end{aligned}
$$

Assume that the solution $u$ of (1.1) is in $\prod_{k=1}^{K} H^{t_{k}}\left(\Omega_{k}\right)$. Then the solution $\left(u^{\delta}, \lambda^{\delta}\right)$ of (1.2) satisfies

$$
\begin{aligned}
\sum_{k=1}^{K}\left\|u-u^{\delta}\right\|_{H^{1}\left(\Omega_{k}\right)}^{2}+\sum_{i=1}^{N}\left\|a \frac{\partial u}{\partial \mathbf{n}_{\gamma_{i}}}-\lambda^{\delta}\right\|_{\left(H_{00}^{\frac{1}{2}}\left(\gamma_{i}\right)\right)^{\prime}}^{2} \\
\lesssim\left[1+\log \left\lceil\max _{1 \leq i \leq N} h_{m(i)}^{\delta} / h_{s(i)}^{\delta}\right]\right]^{2} \sum_{k=1}^{K}\left(h_{k}^{\delta}\right)^{2 t_{k}-2}\|u\|_{H^{t_{k}}\left(\Omega_{k}\right)}^{2}
\end{aligned}
$$


Proof Let us consider (2.5). We have $\left\|w_{i}^{\delta}\right\|_{H_{00}^{\frac{1}{2}}\left(\gamma_{i}\right)} \lesssim\left(\underline{h}_{i}^{\delta}\right)^{-\frac{1}{2}}\left\|w_{i}^{\delta}\right\|_{L^{2}\left(\gamma_{i}\right)}$ for any $w_{i}^{\delta} \in W_{0, i}^{\delta}$. So by interpolation and the reiteration theorem, we have $\left\|w_{i}^{\delta}\right\|_{H_{00}^{\frac{1}{2}\left(\gamma_{i}\right)}} \lesssim\left(\underline{h}_{i}^{\delta}\right)^{-\varepsilon}\left\|w_{i}^{\delta}\right\|_{\left[H_{0}^{1}\left(\gamma_{i}\right), L^{2}\left(\gamma_{i}\right)\right]_{\frac{1}{2}+\varepsilon}}$ for any $w_{i}^{\delta} \in W_{0, i}^{\delta}$ and $\varepsilon \in\left[0, \frac{1}{2}\right]$. Furthermore, by (A.5), Lemma 2.2 and interpolation, we have that $\Pi_{i}^{\delta}:\left[H_{0}^{1}\left(\gamma_{i}\right), L^{2}\left(\gamma_{i}\right)\right]_{\theta} \rightarrow\left[H_{0}^{1}\left(\gamma_{i}\right), L^{2}\left(\gamma_{i}\right)\right]_{\theta}$ is bounded uniformly in $\delta \in \mathcal{I}$ and $\theta \in[0,1]$. So by applying Lemma 2.6 with $\theta=\frac{1}{2}+\varepsilon$, we find that

$$
\begin{aligned}
& \left\|\Pi_{i}^{\delta}\left[u-x^{\delta}\right]_{\gamma_{i}}\right\|_{H_{00}^{\frac{1}{2}}\left(\gamma_{i}\right)} \\
& \quad \lesssim\left(h_{i}^{\delta}\right)^{-\varepsilon} \varepsilon^{-1}\left\{\left\|\left.\left(u-x^{\delta}\right)\right|_{\Omega_{m(i)}}\right\|_{H^{\frac{1}{2}-\varepsilon}\left(\gamma_{i}\right)}+\left\|\left.\left(u-x^{\delta}\right)\right|_{\Omega_{s(i)}}\right\|_{H^{\frac{1}{2}-\varepsilon}\left(\gamma_{i}\right)}\right\} \\
& \quad \lesssim\left(h_{i}^{\delta}\right)^{-\varepsilon} \varepsilon^{-1}\left\{\left\|u-x^{\delta}\right\|_{H^{1-\varepsilon}\left(\Omega_{m(i)}\right)}+\left\|u-x^{\delta}\right\|_{H^{1-\varepsilon}\left(\Omega_{s(i)}\right)}\right\}
\end{aligned}
$$

where, for the last inequality, we have used that, for any $\eta \in\left[0, \frac{1}{2}\right)$, the trace operator is bounded from $H^{1-\varepsilon}\left(\Omega_{k}\right) \rightarrow H^{\frac{1}{2}-\varepsilon}\left(\partial \Omega_{k}\right)$ uniformly in $\varepsilon \in[0, \eta]$. For $\varepsilon \leq \min _{k} t_{k}-1$, a duality argument using $H^{1}\left(\Omega_{k}\right)$ as pivot space shows that $\inf _{x_{k}^{\delta} \in X_{k}^{\delta}}\left\|u-x_{k}^{\delta}\right\|_{H^{1-\varepsilon}\left(\Omega_{k}\right)} \lesssim$ $\left(h_{k}^{\delta}\right)^{t_{k}-1+\varepsilon}\|u\|_{H^{t_{k}\left(\Omega_{k}\right)}}$. With $\zeta:=\left\lceil\max _{1 \leq i \leq N} h_{m(i)}^{\delta} / h_{s(i)}^{\delta}\right\rceil$, from (2.2), (2.4), (2.5), and, to bound the error in the Lagrange multiplier, the trace theorem, we conclude the statement of the theorem with the factor $[1+\log (\zeta)]^{2}$ replaced by $\varepsilon^{-2}\left[1+\zeta^{\varepsilon}\right]^{2}$. By, when $\zeta$ is sufficiently large, taking $\varepsilon=\log (2) / \log (\zeta)$, the proof is completed.

\section{Dual Lagrange multipliers}

\subsection{General framework}

In this subsection, after recalling the definition and the advantages of having dual Lagrange multiplier bases, we sketch a general procedure for their construction. We illustrate various steps of this procedure with an example with $d=2$. Hereafter, in the following subsections, we will give examples with $d=3$ of spaces $X^{\delta}=\prod_{k=1}^{K} X_{k}^{\delta}$ and $M^{\delta}=\prod_{i=1}^{N} M_{i}^{\delta}$ that satisfy all conditions we have imposed, and that can be equipped with such bases.

Let $X_{s(i)}^{\delta}$ and $M_{i}^{\delta}$ be spaces with bases $\left\{x_{s(i), j}^{\delta}: j \in D_{s(i)}^{\delta}\right\}$ and $\left\{m_{i, \ell}^{\delta}: \ell \in I_{i}^{\delta}\right\}$, respectively. We think as the index sets $D_{s(i)}^{\delta}$ and $I_{i}^{\delta}$ as being collections of points, typically 'nodal points', in $\overline{\Omega_{s(i)}}$ and $\overline{\gamma_{i}}$, respectively. Under the natural condition that any linear combination 
of $x_{s(i), j}^{\delta}$ vanishes on $\partial \Omega_{s(i)} \backslash \gamma_{i}$ only if any term vanishes on $\partial \Omega_{s(i)} \backslash \gamma_{i}$, with

$$
S_{0, i}^{\delta}:=\left\{\ell \in D_{s(i)}^{\delta}:\left.x_{s(i), \ell}^{\delta}\right|_{\partial \Omega_{s(i)} \backslash \gamma_{i}}=0,\left.x_{s(i), \ell}^{\delta}\right|_{\gamma_{i}} \not \equiv 0\right\},
$$

the collection $\left\{x_{s(i), \ell}^{\delta} \mid \gamma_{i}: \ell \in S_{0, i}^{\delta}\right\}$ spans $W_{0, i}^{\delta}$.

Our aim is to select bases for $X_{s(i)}^{\delta}$ and $M_{i}^{\delta}$ in such a way that

$I_{i}^{\delta} \subset S_{0, i}^{\delta}$ and

$\left\langle\left. x_{s(i), \ell}^{\delta}\right|_{\gamma_{i}}, m_{i, \ell^{\prime}}^{\delta}\right\rangle_{L^{2}\left(\gamma_{i}\right)} \begin{cases}\approx\left\|\left.x_{s(i), \ell}^{\delta}\right|_{\gamma_{i}}\right\|_{L^{2}\left(\gamma_{i}\right)}\left\|m_{i, \ell^{\prime}}^{\delta}\right\|_{L^{2}\left(\gamma_{i}\right)} & \text { if } \ell=\ell^{\prime} \in I_{i}^{\delta}, \\ =0 & \text { if } \ell \neq \ell^{\prime} \in I_{i}^{\delta},\end{cases}$

i.e., such that, suitably scaled, the collections $\left\{m_{i, \ell}^{\delta}: \ell \in I_{i}^{\delta}\right\}$ and $\left\{x_{s(i), \ell}^{\delta} \mid \gamma_{i}: \ell \in I_{i}^{\delta}\right\}$ are biorthogonal, or dual to each other, explaining why we speak about dual Lagrange multipliers. Dual Lagrange multipliers were introduced earlier, with $I_{i}^{\delta}=S_{0, i}^{\delta}$ and so $\operatorname{dim} M_{i}^{\delta}=\operatorname{dim} W_{0, i}^{\delta}$, see $[27,22,17,28]$. Here we generalize the concept of dual Lagrange multipliers by allowing that $\operatorname{dim} M_{i}^{\delta}<\operatorname{dim} W_{0, i}^{\delta}$.

Under the additional assumption that $\left\{\left.x_{s(i), \ell}^{\delta}\right|_{\gamma_{i}}: \ell \in I_{i}^{\delta}\right\}$ and $\left\{m_{i, \ell}^{\delta}: \ell \in I_{i}^{\delta}\right\}$ are uniformly $L^{2}\left(\gamma_{i}\right)$-stable collections, with which we mean that $\left\|\sum_{\ell \in I_{i}^{\delta}} c_{\ell} x_{s(i), \ell}^{\delta}\left|\gamma_{i}\left\|_{L^{2}\left(\gamma_{i}\right)}^{2} \approx \sum_{\ell \in I_{i}^{\delta}}\left|c_{\ell}\right|^{2}\right\| x_{s(i), \ell}^{\delta}\right|_{\gamma_{i}}\right\|_{L^{2}\left(\gamma_{i}\right)}^{2}$, and analogously for the other collection, the first benefit of having such dual Lagrange multipliers is that their existence implies that of uniformly $L^{2}\left(\gamma_{i}\right)$-bounded mortar projectors. Indeed, from (3.2) it follows that $\beta_{i} \gtrsim 1$, even if in (1.4) we would replace $W_{i, 0}^{\delta}$ by its subspace $\operatorname{span}\left\{\left.x_{s(i), \ell}^{\delta}\right|_{\gamma_{i}}: \ell \in I_{i}^{\delta}\right\}$. by

Secondly, defining for $1 \leq k \leq K$, the set of "free" indices in $\bar{\Omega}_{k}$

$$
\breve{D}_{k}^{\delta}=\left\{j \in D_{k}^{\delta}: j \notin I_{i}^{\delta} \text { for any } 1 \leq i \leq N \text { with } s(i)=k\right\},
$$

the property (3.2) of having dual Lagrange multipliers implies that

$$
\bigcup_{k=1}^{K}\left\{x_{k, j}^{\delta}-\sum_{i=1}^{N} \sum_{\ell \in I_{i}^{\delta}} \frac{b\left(x_{k, j}^{\delta}, m_{i, \ell}^{\delta}\right)}{b\left(x_{s(i), \ell}^{\delta}, m_{i, \ell}^{\delta}\right)} x_{s(i), \ell}^{\delta}: j \in \breve{D}_{k}^{\delta}\right\}
$$

is a basis for $V^{\delta}$. The availability of such bases opens the way to apply efficient multi-level solvers to the elliptic system (1.3). It is generally observed that such schemes are more efficient than iterative solvers for the saddle point formulation (1.2). 
Because of the incorporation of homogeneous Dirichlet boundary conditions at $\partial \gamma_{i}$ in $W_{0, i}^{\delta}$, but not in $M_{i}^{\delta}$, the construction of dual Lagrange multipliers $\left\{m_{i, \ell}^{\delta}: \ell \in I_{i}^{\delta}\right\}$ requires a special treatment near $\partial \gamma_{i}$. In our examples, initially we ignore the interface boundary problems, and construct $\left\{x_{s(i), j}^{\delta}: j \in D_{s(i)}^{\delta}\right\}$ and $\left\{\bar{m}_{i, \ell}^{\delta}: \ell \in \bar{I}_{i}^{\delta}\right\}$, generally with $\bar{I}_{i}^{\delta} \supsetneq I_{i}^{\delta}:=\bar{I}_{i}^{\delta} \cap S_{0, i}^{\delta}$, for which

$\left\langle\left. x_{s(i), \ell}^{\delta}\right|_{\gamma_{i}}, \bar{m}_{i, \ell^{\prime}}^{\delta}\right\rangle_{L^{2}\left(\gamma_{i}\right)}\left\{\begin{array}{lr}\bar{\sim}\left\|\left.x_{s(i), \ell}^{\delta}\right|_{\gamma_{i}}\right\|_{L^{2}\left(\gamma_{i}\right)}\left\|\bar{m}_{i, \ell^{\prime}}^{\delta}\right\|_{L^{2}\left(\gamma_{i}\right)} & \text { if } I_{i}^{\delta} \ni \ell=\ell^{\prime}, \\ =0 & \text { if } I_{i}^{\delta} \ni \ell \neq \ell^{\prime} \in \bar{I}_{i}^{\delta},\end{array}\right.$

and such that $\left\{x_{s(i), \ell}^{\delta} \mid \gamma_{i}: \ell \in I_{i}^{\delta}\right\}$ and $\left\{\bar{m}_{i, \ell}^{\delta}: \ell \in \bar{I}_{i}^{\delta}\right\}$ are uniformly $L^{2}\left(\gamma_{i}\right)$-stable.

Example 3.1 Let $X_{s(i)}^{\delta}$ be the quadratic Lagrange finite element space, incorporating homogeneous Dirichlet boundary conditions on $\partial \Omega \cap$ $\partial \Omega_{s(i)}$, with respect to a conforming, shape regular and locally quasiuniform partition of $\overline{\Omega_{s(i)}} \subset \mathbb{R}^{2}$ into triangles as illustrated in Figure 3.1. Let the auxiliary space $\bar{M}_{i}^{\delta}$ be the linear Lagrange finite element space with respect to the induced partition of $\overline{\gamma_{i}}$ into intervals. With $D_{s(i)}^{\delta}$ being the set of all vertices and midpoints of edges of all triangles not on $\partial \Omega$, and with $\bar{I}_{i}^{\delta}$ the set of all vertices of all intervals, we equip $X_{s(i)}^{\delta}$ and $\bar{M}_{i}^{\delta}$ with standard nodal bases $\left\{x_{s(i), j}^{\delta}: j \in D_{s(i)}^{\delta}\right\}$ and $\left\{\bar{m}_{i, \ell}^{\delta}: \ell \in \bar{I}_{i}^{\delta}\right\}$. Then the set $S_{0, i}^{\delta}$ from (3.1) is the collection of all vertices and midpoints of edges of triangles on $\gamma_{i}$, and $I_{i}^{\delta}:=\bar{I}_{i}^{\delta} \cap S_{0, i}^{\delta}$ is equal to $\bar{I}_{i}^{\delta} \backslash \partial \gamma_{i}$. Since the linear and quadratic functions in the right picture of Figure 3.1 form biorthogonal sets, we conclude that $\left\{\left.x_{s(i), \ell}\right|_{\gamma_{i}}: \ell \in I_{i}^{\delta}\right\}$ and $\left\{\bar{m}_{i, \ell^{\prime}}^{\delta}: \ell \in \bar{I}_{i}^{\delta}\right\}$ satisfy (3.3). Moreover, both collections are uniformly $L^{2}\left(\gamma_{i}\right)$-stable.

We consider $X_{s(i)}^{\delta}$ to be some standard finite element space of degree $r_{s(i)}-1 \geq 1$, incorporating homogeneous Dirichlet boundary conditions on $\partial \Omega \cap \partial \Omega_{s(i)}$, with respect to a shape regular and locally quasi-uniform partition. As a consequence, the conditions (A.3), (A.4) and (2.6) involving $W_{0, i}^{\delta}$ or $X_{s(i)}^{\delta}$ are satisfied. At the multiplier side, assuming that

$$
\frac{d-1}{2}<r_{s(i)}-1,
$$

we will construct a collection of functionals $\left\{\psi_{i, \ell}^{\delta}: \ell \in \bar{I}_{i}^{\delta}\right\} \subset C\left(\gamma_{i}\right)^{\prime}$, with

$$
\left|\psi_{i, \ell}^{\delta}(v)\right| \lesssim\left(\operatorname{diam}\left(\operatorname{supp} \bar{m}_{i, \ell}^{\delta}\right)\right)^{\frac{d-1}{2}}\left\|\bar{m}_{i, \ell}^{\delta}\right\|_{L^{2}\left(\gamma_{i}\right)}^{-1}\|v\|_{L^{\infty}\left(\gamma_{i}\right)},
$$



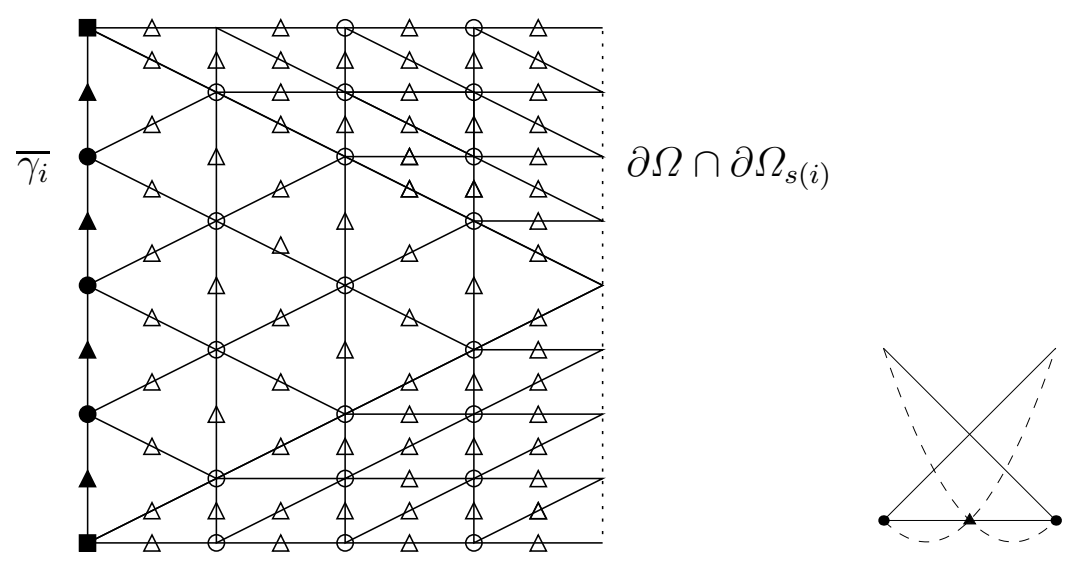

Fig. 3.1: $D_{s(i)}^{\delta}=\{0\} \cup\{\triangle\} \cup\{\bullet\} \cup\{\boldsymbol{\Delta}\} \cup\{\boldsymbol{\square}\}, S_{0, i}^{\delta}=\{\bullet\} \cup\{\boldsymbol{\Delta}\}$, $\bar{I}_{i}^{\delta}=\{\bullet\} \cup\{\boldsymbol{\square}\}, I_{i}^{\delta}=\{\bullet\}$ in the situation of Example 3.1. The linear and quadratic functions in the right picture form biorthogonal sets.

such that

$$
v \mapsto \sum_{\ell \in \bar{I}_{i}^{\delta}} \psi_{i, \ell}^{\delta}(v) \bar{m}_{i, \ell}^{\delta} \text { is a quasi-interpolator of order } r_{s(i)}-2,
$$

meaning that it preserves $P_{r_{s(i)}-2}\left(\gamma_{i}\right)$, where in addition, both the $\bar{m}_{i, \ell}^{\delta}$ and $\psi_{i, \ell}^{\delta}$ are local with respect to the partition underlying $W_{0, i}^{\delta}$.

Remark 3.2 The condition (3.4) can be avoided by constructing smoother $\psi_{i, \ell}^{\delta}$, see $[21, \S 2.1 .1]$.

Example 3.1 (continued) The mapping $v \mapsto \sum_{\ell \in \bar{I}_{i}^{\delta}} v(\ell) \bar{m}_{i, \ell}^{\delta}$ is a valid (quasi-) interpolator of order $r_{s(i)}-2=1$.

In view of (3.2), the remaining problem to be solved is that generally $\bar{I}_{i}^{\delta} \supsetneq I_{i}^{\delta}$. Obviously, we cannot simply remove those $\bar{m}_{i, n}^{\delta}$ from $\left\{\bar{m}_{i, \ell}^{\delta}: \ell \in \bar{I}_{i}^{\delta}\right\}$ with $n \in \bar{I}_{i}^{\delta} \backslash I_{i}^{\delta}$, since generally the span of the remaining set will not have appropriate approximation properties. Therefore, together with the removal of such an $\bar{m}_{i, n}^{\delta}$, we will add suitable multiples of it to some of the remaining $\bar{m}_{i, \ell}^{\delta}$. For some fixed $q \geq \operatorname{dim} P_{r_{s(i)}-2}\left(\gamma_{i}\right)$, for each $n \in \bar{I}_{i}^{\delta} \backslash I_{i}^{\delta}$, we select $\ell_{1}^{n}, \ldots, \ell_{q}^{n} \in I_{i}^{\delta}$ with $\operatorname{dist}\left(\operatorname{supp} \bar{m}_{i, \ell_{r}^{n}}^{\delta}, \operatorname{supp} \bar{m}_{i, n}^{\delta}\right) \lesssim \operatorname{diam}\left(\operatorname{supp} \bar{m}_{i, n}^{\delta}\right)$, such that the problem $\underset{\left(\sigma_{r}^{n}\right)_{1 \leq r \leq q}}{\operatorname{argmin}}\left\{\sum_{r=1}^{q} \frac{\left|\sigma_{r}^{n}\right|^{2}}{\left\|\bar{m}_{i, \ell_{r}^{n}}^{\delta}\right\|_{L^{2}\left(\gamma_{i}\right)}^{2}}: \sum_{r=1}^{q} \sigma_{r}^{n} \psi_{i, \ell_{r}^{n}}^{\delta}(p)=\psi_{i, n}^{\delta}(p), p \in P_{r_{s(i)}-2}\left(\gamma_{i}\right)\right\}$ 
has a solution with $\left|\sigma_{r}^{n}\right| \lesssim\left\|\bar{m}_{i, \ell_{r}^{\prime}}^{\delta}\right\|_{L^{2}\left(\gamma_{i}\right)} /\left\|\bar{m}_{i, n}^{\delta}\right\|_{L^{2}\left(\gamma_{i}\right)}, 1 \leq r \leq q$. In all but one of our examples $q=\operatorname{dim} P_{r_{s(i)}-2}\left(\gamma_{i}\right)$, and the constraints already uniquely determine $\left(\sigma_{r}^{n}\right)_{1 \leq r \leq q}$. We remove $n$ from $\bar{I}_{i}^{\delta}$ and thus $\bar{m}_{i, n}^{\delta}$ from the collection $\left\{\bar{m}_{i, \ell}^{\delta}: \ell \in \bar{I}_{i}^{\delta}\right\}$, and redefine

$$
\bar{m}_{i, \ell_{r}^{n}}^{\delta} \leftarrow \bar{m}_{i, \ell_{r}^{n}}^{\delta}+\sigma_{r}^{n} \bar{m}_{i, n}^{\delta}, \quad 1 \leq r \leq q .
$$

Note that the reduced and modified collection $\left\{\bar{m}_{i, \ell}^{\delta}: \ell \in \bar{I}_{i}^{\delta}\right\}$ still satisfies (3.3). Moreover, since before the removal of $\bar{m}_{i, n}^{\delta}$ and the redefinition of $\bar{m}_{i, \ell_{r}^{n}}^{\delta}$, we had $p=\sum_{\ell \in \bar{I}_{i}^{\delta}} \psi_{i, \ell}^{\delta}(p) m_{i, \ell}^{\delta}$ for any $p \in P_{r_{s(i)}-2}\left(\gamma_{i}\right)$, from (3.7) we conclude that this property is retained. After the removal of all $n \in \bar{I}_{i}^{\delta} \backslash I_{i}^{\delta}$ and the corresponding updates of the $\bar{m}_{i, \ell_{r}^{n}}^{\delta}$, the collection $\left\{\bar{m}_{i, \ell}^{\delta}: \ell \in I_{i}^{\delta}\right\}$ will be written as $\left\{m_{i, \ell}^{\delta}: \ell \in I_{i}^{\delta}\right\}$, and we conclude that we have obtained (3.2), as well as (A.2).

Example 3.1 (continued) With $n$ being one of the points on $\partial \gamma_{i}$, let $\ell_{1}^{n}$, $\ell_{2}^{n}$ be the vertices of the first interval interior to $\gamma_{i}$ next to $n$, assuming that the partition is sufficiently fine such that such an interval exists. Since $2=\operatorname{dim} P_{1}\left(\gamma_{i}\right)$, the equation $\sum_{r=1}^{2} \sigma_{r}^{n} p\left(\ell_{r}^{n}\right)=p(n)$ for $p \in$ $P_{1}\left(\gamma_{i}\right)$ has one solution $\sigma_{1}^{n}=\frac{\ell_{2}^{n}}{\ell_{2}^{n}-\ell_{1}^{n}}$ and $\sigma_{2}^{n}=\frac{\ell_{1}^{n}}{\ell_{1}^{n}-\ell_{2}^{n}}$. The resulting dual Lagrange multipliers are illustrated in Figure 3.2.

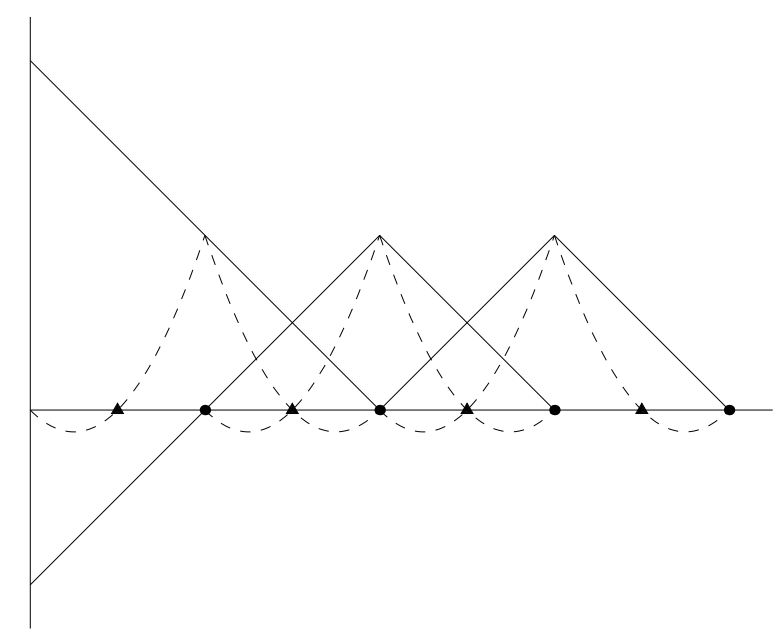

Fig. 3.2: $\left\{m_{i, \ell}^{\delta}: \ell \in I_{i}^{\delta}\right\}$ and $\left\{\left.x_{s(i), \ell}^{\delta}\right|_{\gamma_{i}}: \ell \in I_{i}^{\delta}\right\}$ in the situation of Example 3.1. 
Although the arguments are essentially known, for completeness we verify whether the resulting space $M_{i}^{\delta}:=\operatorname{span}\left\{m_{i, \ell}^{\delta}: \ell \in I_{i}^{\delta}\right\}$ constructed in this way indeed has appropriate approximation properties. For any element $T$ from the underlying partition, by assumption there exists a ball $B(T) \supset T$ with $\operatorname{diam}(B(T)) \lesssim \operatorname{diam}(T)$ such that $\operatorname{supp} \psi_{i, \ell}^{\delta} \subset B(T)$ for any of the uniformly bounded number of $\ell \in I_{i}^{\delta}$ with $\operatorname{supp} m_{i, \ell}^{\delta} \cap T \neq \emptyset$. Using (3.4) and (3.5), by applying the Sobolev embedding theorem on a Lipschitz domain with volume of order 1, as well as $\|\cdot\|_{H^{r_{s(i)}}-1} \approx\left[\|\cdot\|_{L^{2}}^{2}+|\cdot|_{H^{r_{s(i)}-1}}^{2}\right]^{\frac{1}{2}}$ on such a domain, together with a homogeneity argument, we infer that

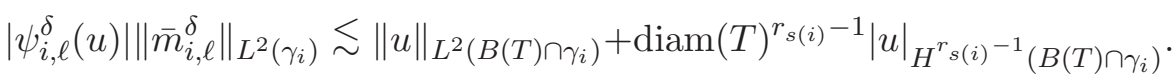

By an application of the Bramble-Hilbert lemma, for $u \in H^{r_{s(i)}-1}\left(\gamma_{i}\right)$ we have

$$
\begin{aligned}
& \left\|u-\sum_{\ell \in I_{i}^{\delta}} \psi_{i, \ell}^{\delta}(u) m_{i, \ell}^{\delta}\right\|_{L^{2}(T)} \\
& =\inf _{p \in P_{r_{s(i)}-2\left(\gamma_{i}\right)}}\left\|u-p-\sum_{\ell \in I_{i}^{\delta}} \psi_{i, \ell}^{\delta}(u-p) m_{i, \ell}^{\delta}\right\|_{L^{2}(T)} \\
& \lesssim \inf _{p \in P_{r_{s(i)}-2\left(\gamma_{i}\right)}}\|u-p\|_{L^{2}\left(B(T) \cap \gamma_{i}\right)}+\operatorname{diam}(T)^{r_{s(i)}-1}|u|_{H^{r_{s(i)}-1}\left(B(T) \cap \gamma_{i}\right)} \\
& \lesssim \operatorname{diam}(T)^{r_{s(i)}-1}|u|_{H^{r_{s(i)}}-1\left(B(T) \cap \gamma_{i}\right)},
\end{aligned}
$$

which yields (2.7).

For the case that the partition underlying $W_{i, 0}^{\delta}$ is not quasi-uniform, the final condition that we have to verify is (A.6), that is, whether the mortar projectors can be selected as being local operators. Suppose we can extend $\left\{m_{i, \ell}^{\delta}: \ell \in I_{i}^{\delta}\right\}$ to a uniformly $L^{2}\left(\gamma_{i}\right)$-stable and local collection $\left\{m_{i, \ell}^{\delta}: \ell \in S_{i, 0}^{\delta}\right\}$ with

$$
\left\langle\left. x_{s(i), \ell}^{\delta}\right|_{\gamma_{i}}, m_{i, \ell^{\prime}}^{\delta}\right\rangle_{L^{2}\left(\gamma_{i}\right)}\left\{\begin{array}{lr}
\approx\left\|\left.x_{s(i), \ell}^{\delta}\right|_{\gamma_{i}}\right\|_{L^{2}\left(\gamma_{i}\right)}\left\|m_{i, \ell^{\prime}}^{\delta}\right\|_{L^{2}\left(\gamma_{i}\right)} & \text { if } S_{i, 0}^{\delta} \ni \ell=\ell^{\prime}, \\
=0 & \text { if } I_{i}^{\delta} \ni \ell \neq \ell^{\prime} \in S_{i, 0}^{\delta} .
\end{array}\right.
$$

Without loss of generality, let us assume that $\left\|\left.x_{s(i), \ell}^{\delta}\right|_{\gamma_{i}}\right\|_{L^{2}\left(\gamma_{i}\right)} \approx 1$ and $\left\|m_{i, \ell^{\prime}}^{\delta}\right\|_{L^{2}\left(\gamma_{i}\right)} \approx 1$. Then, assuming that also $\left\{\left.x_{s(i), \ell}^{\delta}\right|_{\gamma_{i}}: \ell \in S_{i, 0}^{\delta}\right\}$ is uniformly $L^{2}\left(\gamma_{i}\right)$-stable and local, with respect to the partition of $S_{0, i}^{\delta}$ into $I_{i}^{\delta}$ and $S_{0, i}^{\delta} \backslash I_{i}^{\delta}$, the matrix $\mathbf{R}_{i}^{\delta}:=\left[\left\langle\left. x_{i, \ell}^{\delta}\right|_{\gamma_{i}}, m_{i, \ell^{\prime}}^{\delta}\right\rangle_{L^{2}\left(\gamma_{i}\right)}\right]_{\ell, \ell^{\prime} \in S_{0, i}^{\delta}}$

is of the form $\left[\begin{array}{cc}\mathbf{D}_{i, 1}^{\delta} & 0 \\ \mathbf{A}_{i}^{\delta} & \mathbf{D}_{i, 2}^{\delta}\end{array}\right]$, with $\mathbf{D}_{i, 1}^{\delta}, \mathbf{D}_{i, 2}^{\delta}$ being diagonal matrices 
that are uniformly boundedly invertible, and $\mathbf{A}_{i}^{\delta}$ being a uniformly sparse and bounded matrix. We infer that $\mathbf{R}_{i}^{\delta}$ is uniformly boundedly invertible, and that as a consequence

$$
\Pi_{i}^{\delta}:\left.v \mapsto \sum_{\ell \in S_{i, 0}^{\delta}}\left\langle v, m_{i, \ell}^{\delta}\right\rangle \sum_{\ell^{\prime} \in S_{i, 0}^{\delta}}\left(\left(\mathbf{R}_{i}^{\delta}\right)^{-1}\right)_{\ell, \ell^{\prime}} x_{s(i), \ell^{\prime}}^{\delta}\right|_{\gamma_{i}}
$$

is an uniformly $L_{2}\left(\gamma_{i}\right)$-bounded projector onto $W_{i, 0}^{\delta}$ with

$\left\langle\Pi_{i}^{\delta} v, \mu_{i}^{\delta}\right\rangle_{L^{2}\left(\gamma_{i}\right)}=\left\langle v, \mu_{i}^{\delta}\right\rangle_{L^{2}\left(\gamma_{i}\right)} \quad v \in L^{2}\left(\gamma_{i}\right), \mu_{i}^{\delta} \in \operatorname{span}\left\{m_{i, \ell}^{\delta}: \ell \in S_{i, 0}^{\delta}\right\}$, and thus in particular that it is a valid mortar projector. Since this $\Pi_{i}^{\delta}$ is local we conclude that (3.9) implies (A.6).

Remark 3.2 As an alternative for taking $M_{i}^{\delta}=\operatorname{span}\left\{m_{i, \ell}^{\delta}: \ell \in I_{i}^{\delta}\right\}$, one may use the auxiliary functions $m_{i, \ell}^{\delta}$ for $\ell \in S_{i, 0}^{\delta} \backslash I_{i}^{\delta}$ to define $M_{i}^{\delta}=\operatorname{span}\left\{m_{i}^{\delta}: i \in S_{0, i}^{\delta}\right\}$, with which one gets into the situation of having so called quasi-dual Lagrange multipliers as in [19], and $\operatorname{dim} W_{0, i}^{\delta}=\operatorname{dim} M_{i}^{\delta}$. This approach, however, leads to a more complicated coupling between master and slave sides. In the cases where we have tested both approaches, the discretization errors were almost equal.

Example 3.1 (continued) For each $\ell \in S_{i, 0}^{\delta} \backslash I_{i}^{\delta}$, i.e., for each midpoint of any interval in the partition of $\overline{\gamma_{i}}$, with neighbours $\ell_{1}, \ell_{2} \in \bar{I}_{i}^{\delta}$, being the endpoints of the interval, it is easy to find some $m_{i, \ell}^{\delta}$ supported in that interval such that $\left\langle x_{s(i), \ell_{k}}^{\delta} \mid \gamma_{i}, m_{i, \ell}^{\delta}\right\rangle_{L^{2}\left(\gamma_{i}\right)}=0$ for $k=1,2,\left\langle x_{s(i), \ell}^{\delta} \mid \gamma_{i}, m_{i, \ell}^{\delta}\right\rangle_{L^{2}\left(\gamma_{i}\right)} \approx\left\|\left.x_{s(i), \ell}^{\delta}\right|_{\gamma_{i}}\right\|_{L^{2}\left(\gamma_{i}\right)}\left\|m_{i, \ell}^{\delta}\right\|_{L^{2}\left(\gamma_{i}\right)}$ and such that $\left\{m_{i, \ell}^{\delta}: \ell \in S_{i, 0}^{\delta}\right\}$ is an $L^{2}\left(\gamma_{i}\right)$-stable collection.

In first and last example from the next subsection, where we consider possibly non quasi-uniform meshes, the condition (A.6) can be as easily verified as in the above Example 3.1. We will therefore not return to this issue.

Finally in this subsection, we comment on how to work efficiently with the resulting dual Lagrange multipliers. Concerning the solution of (1.3), the basis $\cup_{i=1}^{N}\left\{m_{i, \ell}^{\delta}: \ell \in I_{i}^{\delta}\right\}$ for $M_{i}^{\delta}$ only enters via the matrices

$\mathbf{M}_{i, M}^{\delta}:=\left[b\left(x_{m(i), j}^{\delta}, m_{i, \ell}^{\delta}\right)\right]_{\ell \in I_{i}^{\delta}, j \in \breve{D}_{m(i)}^{\delta}}, \mathbf{M}_{i, S}^{\delta}:=\left[b\left(x_{s(i), j}^{\delta}, m_{i, \ell}^{\delta}\right)\right]_{\ell \in I_{i}^{\delta}, j \in \breve{D}_{s(i)}^{\delta}}$,

and

$$
\mathbf{S}_{i}^{\delta}:=\operatorname{diag}\left[b\left(x_{s(i), \ell}^{\delta}, m_{i, \ell}^{\delta}\right)\right]_{\ell \in I_{i}^{\delta}} .
$$


In view of this, instead of directly working with the basis functions $m_{i, \ell}^{\delta}$ of which those with supports near $\partial \gamma_{i}$ generally, because of the updates (3.8), have larger supports and have "irregular" definitions, it will be more efficient to apply the procedure outlined below. Let $\left\{\bar{m}_{i, \ell}^{\delta}: \ell \in \bar{I}_{i}^{\delta}\right\}$ be the initial collection satisfying (3.3), i.e., before the removal of any of the functions with indices in $\bar{I}_{i}^{\delta} \backslash I_{i}^{\delta}$ and the corresponding updates of $q$ of the remaining functions. Let $\overline{\mathbf{M}}_{i, M}^{\delta}$, $\overline{\mathbf{M}}_{i, S}^{\delta}$ and $\overline{\mathbf{S}}_{i}^{\delta}$ be the matrices defined as $\mathbf{M}_{i, M}^{\delta}, \mathbf{M}_{i, S}^{\delta}$ and $\mathbf{S}_{i}^{\delta}$ with $\left\{m_{i, \ell}^{\delta}: \ell \in I_{i}^{\delta}\right\}$ replaced by $\left\{\bar{m}_{i, \ell}^{\delta}: \ell \in \bar{I}_{i}^{\delta}\right\}$, meaning that these matrices can be assembled via "regular" finite element computations.

The matrix $\mathbf{S}_{i}^{\delta}$ is now simply obtained from $\overline{\mathbf{S}}_{i}^{\delta}$ by deleting all rows and columns with indices from $\bar{I}_{i}^{\delta} \backslash I_{i}^{\delta}$. With $\mathbf{T}_{i}^{\delta}$ being the $\# I_{i}^{\delta} \times \# \bar{I}_{i}^{\delta}$ matrix containing in its $\ell^{\prime}$ th row the coordinates of $m_{i, \ell^{\prime}}^{\delta}$ in terms of $\left\{\bar{m}_{i, \ell}^{\delta}: \ell \in \bar{I}_{i}^{\delta}\right\}$, we have

$$
\mathbf{M}_{i, M}^{\delta}=\mathbf{T}_{i}^{\delta} \overline{\mathbf{M}}_{i, M}^{\delta} \quad \text { and } \quad \mathbf{M}_{i, S}^{\delta}=\mathbf{T}_{i}^{\delta} \overline{\mathbf{M}}_{i, S}^{\delta}
$$

Fixing some numbering of $\bar{I}_{i}^{\delta}$, where indices from $I_{i}^{\delta}$ precede those from $\bar{I}_{i}^{\delta} \backslash I_{i}^{\delta}$, and with $\overline{\mathbf{e}}_{\ell}$ or $\mathbf{e}_{\ell}$ being the standard (column) basis vector of $\mathbb{R}^{\# \bar{I}_{i}^{\delta}}$ or $\mathbb{R}^{\# I_{i}^{\delta}}$, respectively, i.e., $\overline{\mathbf{e}}_{\ell}$ or $\mathbf{e}_{\ell}$ is 1 on the position corresponding to $\ell$ and is zero elsewhere, in view of (3.8) one infers that

$$
\mathbf{T}_{i}^{\delta}=\sum_{\ell \in I_{i}^{\delta}} \mathbf{e}_{\ell} \overline{\mathbf{e}}_{\ell}^{T}+\sum_{n \in \bar{I}_{i}^{\delta} \backslash I_{i}^{\delta}}\left(\sum_{k=1}^{q} \sigma_{r}^{n} \mathbf{e}_{\ell_{r}^{n}}\right) \overline{\mathbf{e}}_{n}^{T},
$$

indicating an efficient way to apply $\mathbf{T}_{i}^{\delta}$.

Having solved $u^{\delta}$ from (1.3), one retrieves $\lambda^{\delta}$ represented in terms of $\cup_{i=1}^{N}\left\{m_{i, \ell}^{\delta}: \ell \in I_{i}^{\delta}\right\}$ by testing the equation $a\left(u^{\delta}, v^{\delta}\right)+b\left(v^{\delta}, \lambda^{\delta}\right)=$ $f\left(v^{\delta}\right)$ for any $v^{\delta}$ of the form $x_{s(i), \ell}$ for $1 \leq i \leq N, \ell \in I_{i}^{\delta}$. By splitting the vector into $N$ parts corresponding to the different interfaces, and by applying $\left(\mathbf{T}_{i}^{\delta}\right)^{T}$ to the part corresponding to $\gamma_{i}$, one obtains a representation of $\lambda^{\delta}$ in terms of $\cup_{i=1}^{N}\left\{\bar{m}_{i, \ell}^{\delta}: \ell \in \bar{I}_{i}^{\delta}\right\}$, which is more convenient for further processing.

\subsection{Examples}

In this subsection we will give three examples of spaces $X^{\delta}=\prod_{k=1}^{K} X_{k}^{\delta}$ and $M^{\delta}=\prod_{i=1}^{N} M_{i}^{\delta}$ that satisfy all conditions we have imposed, and, moreover, that will be equipped with dual Lagrange multiplier bases using the procedure outlined in $§ 3.1$. In our examples 
$\bar{\Omega}=\cup_{k=1}^{K} \overline{\Omega_{k}} \subset \mathbb{R}^{3}$ will be a polyhedron, and thus so are the subdomains $\Omega_{k}$. In case of piecewise tensor product polynomial approximation on the slave domain, dual Lagrange multiplier bases can simply be made by taking tensor products of such bases on one-dimensional interfaces. Our examples concern non-tensor product approximations, where the construction is less straightforward. Examples of dual Lagrange multiplier spaces for mortar finite elements can also be found in $[27,22,29,18]$, and in particular for three dimensional domains, in $[8,30,17]$ dealing with linear tetrahedral elements, and in [19] dealing with quadratic serendipity hexahedral elements. Here, we consider quadratic and cubic tetrahedral elements on possibly locally refined partitions. In [19], we constructed dual Lagrange multipliers for a modified quadratic serendipity hexahedral finite element space that along the interface was enriched with bubble functions. Here, by taking an non-standard multiplier space, it will turn out that the addition of these bubble functions can be avoided. The existence of dual Lagrange multipliers depends as much on the choice of the basis for $X_{s(i)}^{\delta}$ as it does on the basis for $M_{i}^{\delta}$. Apart that we allow that $\operatorname{dim} M_{i}^{\delta}<\operatorname{dim} W_{0, s(i)}^{\delta}$, another key point of our constructions will be that we do not simply resort on the nodal basis for $X_{s(i)}^{\delta}$.

3.2.1 Quadratic tetrahedral elements For an $1 \leq i \leq N$, let $\left(T_{s(i)}^{\delta}\right)_{\delta \in \mathcal{I}}$ be a family of shape regular, locally quasi-uniform and conforming partitions of $\Omega_{s(i)}$ into tetrahedra $T$. Let $D_{s(i), v}^{\delta}$ and $D_{s(i), m}^{\delta}$ denote the set of vertices and midpoints of edges, respectively, which are not on $\partial \Omega$ of $T \in T_{s(i)}^{\delta}$, and let $D_{s(i)}^{\delta}=D_{s(i), v}^{\delta} \cup D_{s(i), m}^{\delta}$. We define $X_{s(i)}^{\delta}=C\left(\Omega_{s(i)}\right) \cap H_{*}^{1}\left(\Omega_{s(i)}\right) \cap \prod_{T \in T_{s(i)}^{\delta}} P_{2}(T)$, i.e., $r_{s(i)}-1=2$, and equip it with the nodal basis $\left\{x_{s(i), j}^{\delta}: j \in D_{s(i)}^{\delta}\right\}$. Note that with these definitions, the set $S_{0, i}^{\delta}$ defined in (3.1) is given by $D_{s(i)}^{\delta} \cap \gamma_{i}$.

For $\delta \in \mathcal{I}$, let $\triangle_{i}^{\delta}$ be the partition of $\gamma_{i}$ into triangles $\triangle$ obtained by intersecting all $T \in T_{s(i)}^{\delta}$ with $\gamma_{i}$, and let $\bar{I}_{i}^{\delta}$ denote the set of all vertices of $\triangle \in \triangle_{i}^{\delta}$. We define $\left\{\bar{m}_{i, \ell}: \ell \in \bar{I}_{i}^{\delta}\right\}$ as the nodal basis for $C\left(\gamma_{i}\right) \cap \prod_{\triangle \in \triangle_{i}^{\delta}} P_{1}(\triangle)$. Now we apply a basis transformation at the primal side. For any $\ell \in I_{i}^{\delta}=\bar{I}_{i}^{\delta} \cap S_{0, i}^{\delta}$, which is equal to $D_{s(i), v}^{\delta} \cap \gamma_{i}$, we redefine

$$
x_{s(i), \ell}^{\delta} \leftarrow x_{s(i), \ell}^{\delta}+\sum_{\left\{\tilde{\ell} \in D_{s(i), m}^{\delta}: \ell, \tilde{\ell} \text { are on the same edge of some } \triangle \in \triangle_{i}^{\delta}\right\}}^{\frac{1}{12} x_{s(i) \tilde{\ell} \cdot}^{\delta}}
$$


Then, as we will verify below, we have

$$
\left\langle\left. x_{s(i), \ell}^{\delta}\right|_{\gamma_{i}}, \bar{m}_{i, \ell^{\prime}}^{\delta}\right\rangle_{L^{2}\left(\gamma_{i}\right)}\left\{\begin{array}{lr}
\bar{\sim}\left\|x_{s(i), \ell}^{\delta} \mid \gamma_{i}\right\|_{L^{2}\left(\gamma_{i}\right)}\left\|\bar{m}_{i, \ell^{\prime}}^{\delta}\right\|_{L^{2}\left(\gamma_{i}\right)} & \text { if } I_{i}^{\delta} \ni \ell=\ell^{\prime}, \\
=0 & \text { if } I_{i}^{\delta} \ni \ell \neq \ell^{\prime} \in \bar{I}_{i}^{\delta},
\end{array}\right.
$$

i.e., (3.3) is valid. Obviously $\left\{\bar{m}_{i, \ell}^{\delta}: \ell \in \bar{I}_{i}^{\delta}\right\}$ and $\left\{\left.x_{s(i), \ell}^{\delta}\right|_{\gamma_{i}}: \ell \in I_{i}^{\delta}\right\}$ are local, uniformly $L^{2}\left(\gamma_{i}\right)$-stable collections.

The mapping $v \mapsto \sum_{\ell \in \bar{I}_{i}^{\delta}} v(\ell) \bar{m}_{i, \ell}^{\delta}$ is a (quasi)-interpolator of order $r_{s(i)}-2=1$ that satisfies (3.5).

The general procedure outlined in $\S 3.1$ to remove the degrees of freedom associated to $n \in \bar{I}_{i}^{\delta} \backslash I_{i}^{\delta}$, while retaining the local approximation properties may read as follows: For any vertex $n$ of a $\triangle \in \triangle_{i}^{\delta}$ that is on $\partial \gamma_{i}$, select a $\tilde{\triangle} \in \triangle_{i}^{\delta}$ that has empty intersection with $\partial \gamma_{i}$ and $\operatorname{dist}(\triangle, \tilde{\triangle}) \lesssim \operatorname{diam}(\triangle)$. With $\ell_{1}^{n}, \ell_{2}^{n}, \ell_{3}^{n} \in I_{i}^{\delta}$ being the vertices of $\tilde{\triangle}$, by equipping $P_{1}\left(\gamma_{i}\right)$ with the basis $\left\{p_{1}, p_{2}, p_{3}\right\}$ defined by $p_{r^{\prime}}\left(\ell_{r}^{n}\right)=\delta_{r^{\prime} r}$, one infers that the equation $\sum_{r=1}^{3} \sigma_{r}^{n} p\left(\ell_{r}^{n}\right)=p(n)$ for $p \in P_{1}\left(\gamma_{i}\right)$ has solution $\left(\sigma_{1}^{n}, \sigma_{1}^{n}, \sigma_{1}^{n}\right)$ being the barycentric coordinates of $n$ with respect to $\ell_{1}^{n}, \ell_{2}^{n}, \ell_{3}^{n}$, which are uniformly bounded as required. Now remove $\bar{m}_{i, n}^{\delta}$ from the collection $\left\{\bar{m}_{i, \ell}^{\delta}: \ell \in \bar{I}_{i}^{\delta}\right\}$, and update $\bar{m}_{i, \ell_{r}^{n}} \leftarrow \bar{m}_{i, \ell_{r}^{n}}+\sigma_{r}^{n} \bar{m}_{i, n}^{\delta}, 1 \leq r \leq 3$. After the removal of all $n \in \bar{I}_{i}^{\delta} \backslash I_{i}^{\delta}$, we end up with a set $\left\{m_{i, \ell}^{\delta}: \ell \in I_{i}^{\delta}\right\}$ of dual Lagrange multipliers, which spans a space $M_{i}^{\delta}$ that has the required local approximation properties.

To show (3.11), let $\phi_{r}^{l}, r=1, \cdots, 3$ and $\phi_{r}^{q}, r=1, \cdots, 6$ be the linear and quadratic finite element nodal basis functions on a reference triangle $\hat{\triangle}$ with $\operatorname{vol}(\hat{\triangle})=1$, respectively, using a numbering of the points as in see Figure 3.3. A direct computation gives that

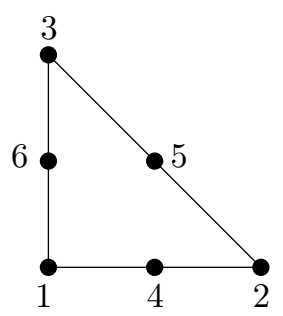

Fig. 3.3: Numbering of the vertices and midpoints of edges on the reference triangle. 


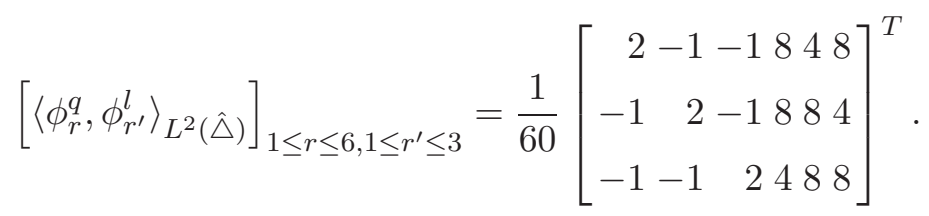

Hence, if we define

$$
\tilde{\phi}_{1}^{q}:=\phi_{1}^{q}+\frac{1}{12}\left(\phi_{4}^{q}+\phi_{6}^{q}\right),
$$

and similarly $\tilde{\phi}_{2}^{q}$ and $\tilde{\phi}_{3}^{q}$ using symmetry in the barycentric coordinates, we obtain

$$
\left[\left\langle\tilde{\phi}_{r}^{q}, \phi_{r^{\prime}}^{l}\right\rangle_{L^{2}(\hat{\Delta})}\right]_{1 \leq r, r^{\prime} \leq 3}=\frac{1}{18} I,
$$

which, in view of the definition (3.10), by using affine bijections between the reference triangle $\hat{\triangle}$ and all $\triangle \in \triangle_{i}^{\delta}$ easily leads to (3.11). The basis transformation (3.10) was made as a result of the above construction of the basis transformation on the reference triangle. Yet, note that not all local basis transformations give rise to corresponding global basis transformations. Essential properties of the modified basis functions on the reference triangle are symmetry in the barycentric coordinates, and the fact that a basis function associated to a point, here a vertex or a midpoint of an edge, vanishes on all edges that do not contain that point.

3.2.2 Quadratic serendipity hexahedral elements For an $1 \leq i \leq N$, let $\left(P_{s(i)}^{\delta}\right)_{\delta \in \mathcal{I}}$ be a family of shape regular, and conforming partitions of $\Omega_{s(i)}$ into parallelepipeds $P$. So here, in order not to be forced to handle cases with hanging nodes, we restrict ourselves to quasiuniform partitions. Let $D_{s(i), v}^{\delta}$ and $D_{s(i), m}^{\delta}$ denote the set of vertices and midpoints of edges, respectively, which are not on $\partial \Omega$ of $P \in$ $P_{s(i)}^{\delta}$, and let $D_{s(i)}^{\delta}=D_{s(i), v}^{\delta} \cup D_{s(i), m}^{\delta}$. We define $X_{s(i)}^{\delta}$ as the quadratic serendipity finite element space with respect to $P_{s(i)}^{\delta}$, i.e., $r_{s(i)}-1=2$, with zero boundary conditions on $\partial \Omega_{s(i)} \cap \partial \Omega$, and equip it with the nodal basis $\left\{x_{s(i), j}^{\delta}: j \in D_{s(i)}^{\delta}\right\}$. Note that the set $S_{0, i}^{\delta}$ is given by $D_{s(i)}^{\delta} \cap \gamma_{i}$.

For $\delta \in \mathcal{I}$, let $\square_{i}^{\delta}$ be the partition of $\gamma_{i}$ into parallelograms obtained by intersecting all $P \in P_{s(i)}^{\delta}$ with $\gamma_{i}$, and let $\bar{I}_{i}^{\delta}$ denote the set of all vertices of $\square \in \square_{i}^{\delta}$. We apply the following basis transformation 
at the primal side. For any $\ell \in I_{i}^{\delta}=\bar{I}_{i}^{\delta} \cap S_{0, i}^{\delta}$, which is equal to $D_{s(i), v}^{\delta} \cap \gamma_{i}$, we redefine

$$
x_{s(i), \ell}^{\delta} \leftarrow x_{s(i), \ell}^{\delta}+\sum_{\left\{\tilde{\ell} \in D_{s(i), m}^{\delta}: \ell, \tilde{\ell} \text { are on the same edge of some } \square \in \square_{i}^{\delta}\right\}}^{\frac{1}{5}} x_{s(i), \tilde{\ell} \cdot}^{\delta}
$$

We will construct an $L^{2}\left(\gamma_{i}\right)$-stable collection $\left\{\bar{m}_{i, \ell}: \ell \in \bar{I}_{i}^{\delta}\right\}$ of local functions such that

$$
\left\langle\left. x_{s(i), \ell}^{\delta}\right|_{\gamma_{i}}, \bar{m}_{i, \ell^{\prime}}^{\delta}\right\rangle_{L^{2}\left(\gamma_{i}\right)}\left\{\begin{array}{lr}
\approx\left\|\left.x_{s(i), \ell}^{\delta}\right|_{\gamma_{i}}\right\|_{L^{2}\left(\gamma_{i}\right)}\left\|\bar{m}_{i, \ell^{\prime}}^{\delta}\right\|_{L^{2}\left(\gamma_{i}\right)} \text { if } I_{i}^{\delta} \ni \ell=\ell^{\prime}, \\
=0 & \text { if } I_{i}^{\delta} \ni \ell \neq \ell^{\prime} \in \bar{I}_{i}^{\delta},
\end{array}\right.
$$

i.e., such that (3.3) is valid. Obviously $\left\{\left.x_{s(i), \ell}^{\delta}\right|_{\gamma_{i}}: \ell \in I_{i}^{\delta}\right\}$ is a local and uniformly $L^{2}\left(\gamma_{i}\right)$-stable collection.

To construct $\left\{\bar{m}_{i, \ell}: \ell \in \bar{I}_{i}^{\delta}\right\}$, using the numbering of the points as in Figure 3.4, let $\phi_{r}^{b}$ for $r=1, \cdots, 4$, and $\phi_{i}^{s}$ for $i=1, \cdots, 8$ denote the bilinear and serendipity finite element nodal basis functions on the reference square $\hat{\square}=(0,1)^{2}$, respectively. A direct computation

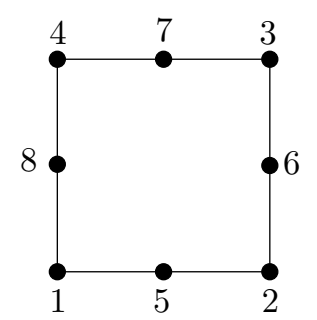

Fig. 3.4: Numbering of the vertices and midpoints of edges on the reference square.

gives that

$$
\left[\left\langle\phi_{r}^{s}, \phi_{1}^{b}\right\rangle_{L^{2}(\hat{\square})}\right]_{1 \leq r \leq 8}=\frac{1}{36}[0-1-1-142224]^{T},
$$

where the other entries follow using symmetry. Because of the missing interior degree of freedom, for the serendipity element we are not able to construct a biorthogonal system by only making a transformation at the 'primal' side. Therefore, for some $\alpha \neq 1$, we set

$$
\tilde{\phi}_{1}^{b}:=\frac{\phi_{1}^{b}+\alpha\left(\phi_{3}^{b}-\phi_{2}^{b}-\phi_{4}^{b}\right)}{1-\alpha},
$$


and define $\tilde{\phi}_{2}^{b}, \tilde{\phi}_{3}^{b}$ and $\tilde{\phi}_{4}^{b}$ analogously. The corresponding collection of global functions $\left\{\bar{m}_{i, \ell}^{\delta}: \ell \in \bar{I}_{i}^{\delta}\right\}$ is now formally defined by $\bar{m}_{i, \ell}^{\delta} \mid \square=$ $\tilde{\phi}_{1}^{b} \circ F_{\square}^{-1}$ for any $\square \in \square_{i}^{\delta}$ that contains $\ell$ as a vertex, and where $F_{\square}$ is an affine bijection between $\hat{\square}$ and $\square$ mapping point 1 onto $\ell$, and $\bar{m}_{i, \ell}$ is zero elsewhere. Note that the $\bar{m}_{i, \ell}^{\delta}$ are discontinuous, and that $\left\{\bar{m}_{i, \ell}^{\delta}: \ell \in \bar{I}_{i}^{\delta}\right\}$ does not span the space of all continuous piecewise bilinears with respect to $\square_{i}^{\delta}$. Yet, although $v \mapsto \sum_{i \in \bar{I}_{i}^{\delta}} v(\ell) \bar{m}_{i, \ell}^{\delta}$ does not preserve $Q_{1}\left(\gamma_{i}\right)$, since $\tilde{\phi}_{1}^{b}+\tilde{\phi}_{2}^{b}=\phi_{1}^{b}+\phi_{2}^{b}, \tilde{\phi}_{2}^{b}+\tilde{\phi}_{3}^{b}=\phi_{2}^{b}+\phi_{3}^{b}$, $\tilde{\phi}_{3}^{b}+\tilde{\phi}_{4}^{b}=\phi_{3}^{b}+\phi_{4}^{b}$, and $\tilde{\phi}_{1}^{b}+\tilde{\phi}_{2}^{b}+\tilde{\phi}_{3}^{b}+\tilde{\phi}_{4}^{b}=\phi_{1}^{b}+\phi_{2}^{b}+\phi_{3}^{b}+\phi_{4}^{b}$ it does preserve $P_{1}\left(\gamma_{i}\right)$, i.e., it is a quasi-interpolator of order $r_{s(i)}-2=1$.

Defining, for some $\theta$,

$$
\tilde{\phi}_{1}^{s}:=\phi_{1}^{s}+\theta\left(\phi_{5}^{s}+\phi_{8}^{s}\right),
$$

and analogously $\tilde{\phi}_{2}^{s}, \tilde{\phi}_{3}^{s}$ and $\tilde{\phi}_{4}^{s}$, a direct computation shows that $\left[\left\langle\tilde{\phi}_{r}^{s}, \tilde{\phi}_{r^{\prime}}^{b}\right\rangle_{L^{2}(\tilde{\square})}\right]_{1 \leq r, r^{\prime} \leq 4}$ is diagonal if and only if $\alpha=\frac{1}{6}$ and $\theta=\frac{1}{5}$, in which case this matrix is equal to $\frac{1}{20} I$. With $\alpha=\frac{1}{6}$ substituted into the definition of $\left\{\bar{m}_{i, \ell}^{\delta}: \ell \in \bar{I}_{i}^{\delta}\right\}$, we conclude (3.13).

The procedure outlined in $\S 3.1$ to remove the degrees of freedom associated to $n \in \bar{I}_{i}^{\delta} \backslash I_{i}^{\delta}$ reads here as follows: For any vertex $n$ of a $\square \in \square_{i}^{\delta}$ that is on $\partial \gamma_{i}$, select a $\tilde{\square} \in \square_{i}^{\delta}$ that has empty intersection with $\partial \gamma_{i}$ and $\operatorname{dist}(\square, \tilde{\square}) \lesssim \operatorname{diam}(\square)$. Remove $n$ from $\bar{I}_{i}^{\delta}$ and thus $\bar{m}_{i, \ell}^{\delta}$ from $\left\{\bar{m}_{i, \ell}^{\delta}: \ell \in \bar{I}_{i}^{\delta}\right\}$, and with $\left(\sigma_{1}^{n}, \sigma_{2}^{n}, \sigma_{3}^{n}\right)$ being the barycentric coordinates of $n$ with respect to three vertices $\ell_{1}^{n}, \ell_{2}^{n}, \ell_{3}^{n}$ of $\tilde{\square}$, update $\bar{m}_{\ell_{r}}^{\delta} \leftarrow \bar{m}_{\ell_{r}}^{\delta}+\sigma_{r}^{n} \bar{m}_{n}^{\delta}, 1 \leq r \leq 3$. After the removal of all $n \in \bar{I}_{i}^{\delta} \backslash I_{i}^{\delta}$, we end up with a set $\left\{m_{i, \ell}^{\delta}: \ell \in I_{i}^{\delta}\right\}$ of dual Lagrange multipliers, which spans a space $M_{i}^{\delta}$ that has the required local approximation properties.

3.2.3 Cubic tetrahedral elements For an $1 \leq i \leq N$, let $\left(T_{s(i)}^{\delta}\right)_{\delta \in \mathcal{I}}$ and $\left(\triangle_{i}^{\delta}\right)_{\delta \in \mathcal{I}}$ be families as in $\S 3.2 .1$ of partitions of $\Omega_{s(i)}$ into tetrahedra, and of $\gamma_{i}$ into triangles, respectively. We define $X_{s(i)}^{\delta}=C\left(\Omega_{s(i)}\right) \cap$ $H_{*}^{1}\left(\Omega_{s(i)}\right) \cap \prod_{T \in T_{s(i)}^{\delta}} P_{3}(T)$, and $\bar{M}_{i}^{\delta}=C\left(\gamma_{i}\right) \cap \prod_{\triangle \in \triangle_{i}^{\delta}} P_{2}(\triangle)$, i.e., $r_{s(i)}-1=3$.

To define a basis $\left\{\bar{m}_{i, \ell}^{\delta}: \ell \in \bar{I}_{i}^{\delta}\right\}$ for $\bar{M}_{i}^{\delta}$, and a modification of the nodal basis $\left\{x_{s(i), j}^{\delta}: j \in D_{s(i)}^{\delta}\right\}$ for $X_{s(i)}^{\delta}$, which only affects $\left\{\left.x_{s(i), \ell}^{\delta}\right|_{\gamma_{i}}: \ell \in S_{0, i}^{\delta}\right\}$, it is sufficient to consider a reference triangle $\hat{\triangle}$ with $\operatorname{vol}(\hat{\triangle})=1$. Using a numbering of the nodal points as indicated 

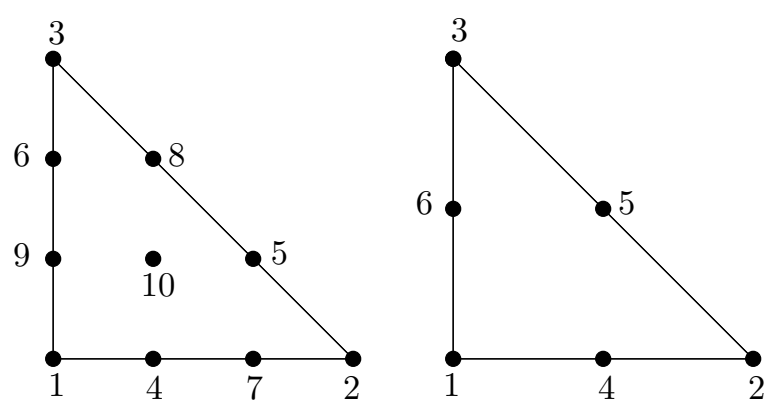

Fig. 3.5: Numbering of nodal points at primal and multiplier side for the cubic tetrahedral case.

in Figure 3.5, let $\left\{\phi_{1}^{c}, \ldots, \phi_{10}^{c}\right\}$ and $\left\{\phi_{1}^{q}, \ldots, \phi_{6}^{q}\right\}$ be the nodal bases for $P_{3}(\hat{\triangle})$ and $P_{2}(\hat{\triangle})$, respectively. We define transformed bases by

$$
\begin{aligned}
& \tilde{\phi}_{1}^{c}=60 \phi_{1}^{c}+\frac{20}{9}\left(\phi_{4}^{c}+\phi_{9}^{c}-\phi_{6}^{c}-\phi_{7}^{c}-\phi_{10}^{c}\right), \\
& \tilde{\phi}_{4}^{c}=10\left(\phi_{4}^{c}+\phi_{7}^{c}\right)-\frac{5}{6} \phi_{10}^{c}, \quad \tilde{\phi}_{10}^{c}=\phi_{10}^{c}, \quad \tilde{\phi}_{7}^{c}=\phi_{4}^{c}-\phi_{7}^{c},
\end{aligned}
$$

and

$$
\tilde{\phi}_{1}^{q}=\phi_{1}^{q}-\frac{1}{8}\left(\phi_{4}^{q}+\phi_{6}^{q}\right), \quad \tilde{\phi}_{4}^{q}=\phi_{4}^{q},
$$

and the others by permuting barycentric coordinates. All these basis functions at primal and multiplier side can be naturally associated to either a vertex, an edge (at the primal side two at each edge) or the center of $\hat{\triangle}$, where they vanish on the opposite edge, on the other two edges, or on all edges, respectively, and finally, where they are either symmetric or anti-symmetric in permutations of the barycentric coordinates. A direct computation shows that

$$
\left[\left\langle\tilde{\phi}_{r}^{c}, \tilde{\phi}_{r^{\prime}}^{q}\right\rangle_{L^{2}(\hat{\Delta})}\right]_{1 \leq r, r^{\prime} \leq 6}=\left[\begin{array}{rrr|r}
\multicolumn{1}{c|}{I} & 0 \\
\hline 0 & 0 & -\frac{1}{8} & \\
-\frac{1}{8} & 0 & 0 & I \\
0 & -\frac{1}{8} & 0 &
\end{array}\right] .
$$

Although this matrix is thus not diagonal, the fact that, with respect to a splitting of the degrees of freedom into those associated to vertices and those associated to edges, it is block lower triangular with diagonal blocks equal to identity matrices which will allow for the construction of local dual Lagrange multiplier bases. 
By lifting $\left\{\tilde{\phi}_{1}^{c}, \ldots, \tilde{\phi}_{10}^{c}\right\}$ and $\left\{\tilde{\phi}_{1}^{q}, \ldots, \tilde{\phi}_{6}^{q}\right\}$ to all $\triangle \in \triangle_{i}^{\delta}$ using affine bijections, and by gluing them continuously over the interfaces, and, at the primal side, by leaving out those functions associated to vertices or edges on $\partial \gamma_{i}$, we end up with the modified collection $\left\{\left.x_{s(i), \ell}^{\delta}\right|_{\gamma_{i}}: \ell \in S_{0, i}^{\delta}\right\}$, and a basis $\left\{\breve{m}_{i, \ell}^{\delta}: \ell \in \bar{I}_{i}^{\delta}\right\}$ for $\bar{M}_{i}^{\delta}$. Here as a natural index set $\bar{I}_{i}^{\delta}$, we use the union of the sets $\bar{I}_{i, v}^{\delta}$ and $\bar{I}_{i, m}^{\delta}$ of all vertices and midpoints of edges of $\triangle \in \triangle_{i}^{\delta}$, respectively. Defining $I_{i, v}^{\delta}=\bar{I}_{i, v}^{\delta} \cap \gamma_{i}, I_{i, m}^{\delta}=\bar{I}_{i, m}^{\delta} \cap \gamma_{i}$ and $I_{i}^{\delta}=I_{i, v}^{\delta} \cup I_{i, m}^{\delta}$, we write $S_{0, i}^{\delta}$ as $I_{i, v}^{\delta} \cup I_{i, m}^{\delta} \cup\left(S_{0, i}^{\delta} \backslash I_{i}^{\delta}\right)$, where $I_{i, v}^{\delta}$ and $I_{i, m}^{\delta}$ are the index sets for those $\left.x_{s(i), \ell}^{\delta}\right|_{\gamma_{i}}$ resulting from the functions $\tilde{\phi}_{1}^{c}, \tilde{\phi}_{2}^{c}, \tilde{\phi}_{3}^{c}$ and $\tilde{\phi}_{4}^{c}, \tilde{\phi}_{5}^{c}, \tilde{\phi}_{6}^{c}$, respectively, and where $S_{0, i}^{\delta} \backslash I_{i}^{\delta}$ is some index set for those $\left.x_{s(i), \ell}^{\delta}\right|_{\gamma_{i}}$ resulting from the functions $\tilde{\phi}_{7}^{c}, \tilde{\phi}_{8}^{c}, \tilde{\phi}_{9}^{c}, \tilde{\phi}_{10}^{c}$.

For $\ell \in I_{i}^{\delta}$, we have

$$
\left\langle\left. x_{s(i), \ell}^{\delta}\right|_{\gamma_{i}}, \breve{m}_{i, \ell}^{\delta}\right\rangle_{L^{2}\left(\gamma_{i}\right)}=d_{\ell}^{\delta}:=\sum_{\left\{\triangle \in \triangle_{i}^{\delta}: \triangle \ni \ell\right\}} \operatorname{vol}(\triangle),
$$

and for any $\ell^{\prime} \in \bar{I}_{i, v}^{\delta}$, and

$$
\begin{aligned}
\ell \in \gamma_{i} \cap \bar{I}_{i, \text { opp }}^{\delta}\left(\ell^{\prime}\right):=\left\{\ell \in I_{i, m}^{\delta}:\right. & : \triangle \triangle \in \triangle_{i}^{\delta} \text { with } \ell^{\prime}, \ell \in \triangle \\
& \text { and } \left.\ell \text { is on the edge opposite to } \ell^{\prime}\right\},
\end{aligned}
$$

see Figure 3.6, we have

$$
\left\langle\left. x_{s(i), \ell}^{\delta}\right|_{\gamma_{i}}, \breve{m}_{i, \ell^{\prime}}^{\delta}\right\rangle_{L^{2}\left(\gamma_{i}\right)}=-\frac{1}{8} \operatorname{vol}\left(\triangle_{\ell, \ell^{\prime}}\right),
$$

whereas $\left\langle x_{s(i), \ell}^{\delta} \mid \gamma_{i}, \breve{m}_{i, \ell^{\prime}}^{\delta}\right\rangle_{L^{2}\left(\gamma_{i}\right)}=0$ for all other $\ell \in I_{i}^{\delta}, \ell^{\prime} \in \bar{I}_{i}^{\delta}$.

Defining the transformed basis $\left\{\bar{m}_{i, \ell}^{\delta}: \ell \in \bar{I}_{i}^{\delta}\right\}$ by

$$
\bar{m}_{i, \ell^{\prime}}^{\delta}:= \begin{cases}\breve{m}_{i, \ell^{\prime}}^{\delta}+\sum_{\ell \in \bar{I}_{i, \mathrm{opp}}^{\delta}\left(\ell^{\prime}\right)} \frac{\frac{1}{8} \operatorname{vol}\left(\triangle_{\ell, \ell^{\prime}}\right)}{d_{\ell}^{\delta}} \breve{m}_{i, \ell}^{\delta} \text { if } \ell^{\prime} \in \bar{I}_{i, v}^{\delta}, \\ \breve{m}_{i, \ell^{\prime}}^{\delta} & \text { if } \ell^{\prime} \in \bar{I}_{i, m}^{\delta},\end{cases}
$$

we find

$$
\left\langle\left. x_{s(i), \ell}^{\delta}\right|_{\gamma_{i}}, \bar{m}_{i, \ell^{\prime}}^{\delta}\right\rangle_{L^{2}\left(\gamma_{i}\right)}= \begin{cases}d_{\ell}^{\delta} & \text { if } I_{i}^{\delta} \ni \ell=\ell^{\prime}, \\ 0 & \text { if } I_{i}^{\delta} \ni \ell \neq \ell^{\prime} \in \bar{I}_{i}^{\delta},\end{cases}
$$

so that (3.3) is valid by $d_{\ell}^{\delta} \approx\left\|\left.x_{s(i), \ell}^{\delta}\right|_{\gamma_{i}}\right\|_{L^{2}\left(\gamma_{i}\right)}\left\|\bar{m}_{i, \ell}^{\delta}\right\|_{L^{2}\left(\gamma_{i}\right)}$.

Concerning implementation, since for $\ell \in \bar{I}_{i, v}^{\delta}, \bar{m}_{i, \ell}^{\delta}$ has a much larger support than $\breve{m}_{i, \ell}^{\delta}$, instead of computing the matrices $\overline{\mathbf{M}}_{i, M}^{\delta}$ and $\overline{\mathbf{M}}_{i, S}^{\delta}$ mentioned in $\S 3.1$, it is much more efficient to write them 
as the composition of the basis transformation defined by (3.15), that can be applied at low cost, and the matrices $\breve{\mathbf{M}}_{i, M}^{\delta}$ and $\breve{\mathbf{M}}_{i, S}^{\delta}$ one gets by replacing $\left\{\bar{m}_{i, \ell}^{\delta}: \ell \in \bar{I}_{i}^{\delta}\right\}$ by $\left\{\breve{m}_{i, \ell}^{\delta}: \ell \in \bar{I}_{i}^{\delta}\right\}$.

Finally, we have to remove $\bar{m}_{i, \ell}^{\delta}$ for $\ell \in \partial \gamma_{i}$. Let $\left\{\bar{n}_{i, \ell}^{\delta}: \ell \in \bar{I}_{i}^{\delta}\right\}$ be the nodal basis for $\bar{M}_{i}^{\delta}$, and let $\breve{\psi}_{i, \ell}^{\delta}(u)=u(\ell)\left(\ell \in \bar{I}_{i}^{\delta}\right)$. With, for $\ell \in \bar{I}_{i, m}^{\delta}, \bar{I}_{i, \text { adj }}^{\delta}(\ell):=\left\{\ell^{\prime} \in \bar{I}_{i, v}^{\delta}: \ell, \ell^{\prime}\right.$ are on one edge of a $\left.\triangle \in \triangle_{i}^{\delta}\right\}$, see Figure 3.6, we define

$\psi_{i, \ell}^{\delta}= \begin{cases}\breve{\psi}_{i, \ell}^{\delta} & \text { if } \ell \in \bar{I}_{i, v}^{\delta}, \\ \breve{\psi}_{i, \ell}^{\delta}+\frac{1}{8} \sum_{\ell^{\prime} \in \bar{I}_{i, \text { adj }}^{\delta}(\ell)} \breve{\psi}_{i, \ell^{\prime}}^{\delta}-\sum_{\left\{\ell^{\prime} \in \bar{I}_{i, v}^{\delta}: \ell \in \bar{I}_{i, \text { opp }}^{\delta}\right.} \frac{\left.\frac{1}{8} \operatorname{vol}\left(\ell_{\ell, \ell^{\prime}}\right)\right\}}{d_{\ell}^{\delta}} \breve{\psi}_{i, \ell^{\prime}}^{\delta} \text { if } \ell \in \bar{I}_{i, m}^{\delta} .\end{cases}$

From (3.14) and (3.15), we infer that

$$
v \mapsto \sum_{\ell \in \bar{I}_{i}^{\delta}} \psi_{i, \ell}^{\delta}(v) \bar{m}_{i, \ell}^{\delta}=\sum_{\ell \in \bar{I}_{i}^{\delta}} \breve{\psi}_{i, \ell}^{\delta}(v) \bar{n}_{i, \ell}^{\delta},
$$

which is thus a quasi-interpolator of order $r_{s(i)}-2=2$.

Now for any $n \in \bar{I}_{i}^{\delta} \backslash I_{i}^{\delta}$, vertex or midpoint of a $\triangle \in \triangle_{i}^{\delta}$, we select a $\tilde{\triangle} \in \triangle_{i}^{\delta}$ with $\operatorname{dist}(\triangle, \tilde{\triangle}) \lesssim \operatorname{diam}(\triangle)$, such that

$$
\bar{I}_{i}^{\delta}(\tilde{\triangle}):=\left(\bar{I}_{i}^{\delta} \cap \tilde{\triangle}\right) \cup \cup_{\ell \in \bar{I}_{i, m}^{\delta} \cap \tilde{\triangle}}\left\{\ell^{\prime} \in \bar{I}_{i, v}^{\delta}: \ell \in \bar{I}_{i, \mathrm{opp}}^{\delta}\left(\ell^{\prime}\right)\right\} \subset \gamma_{i},
$$

see Figure 3.6. Using that, because of the locally quasi-uniform parti-
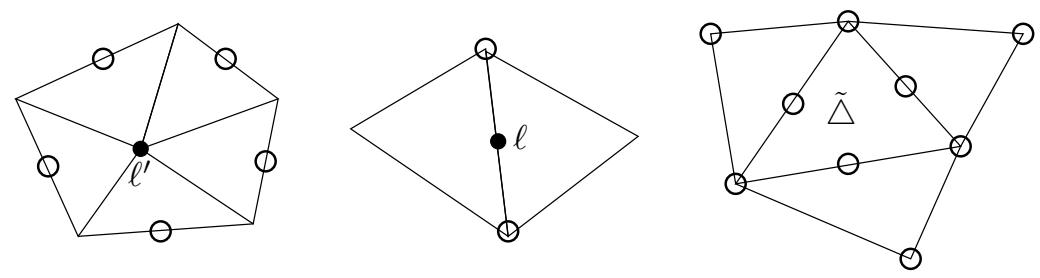

Fig. 3.6: $\bar{I}_{i, \text { opp }}^{\delta}\left(\ell^{\prime}\right), \bar{I}_{i, \text { adj }}^{\delta}(\ell)$ and $\bar{I}_{i}^{\delta}(\tilde{\triangle})$

tion, all $\left\|\bar{m}_{i, \ell}^{\delta}\right\|_{L^{2}\left(\gamma_{i}\right)}$ for $\ell \in I_{i}^{\delta}(\tilde{\triangle})$ are comparable, we solve $\left(\sigma_{\ell}^{n}\right)_{\ell \in \bar{I}_{i}^{\delta}(\tilde{\Delta})}$ from

$\underset{\left(\sigma_{\ell}^{n}\right)_{\ell \in \bar{I}_{i}^{\delta}(\tilde{\Delta})}}{\operatorname{argmin}}\left\{\sum_{\ell \in \bar{I}_{i}^{\delta}(\tilde{\Delta})}\left|\sigma_{\ell}^{n}\right|^{2}: \sum_{\ell \in \bar{I}_{i}^{\delta}(\tilde{\Delta})} \sigma_{\ell}^{n} \psi_{i, \ell}^{\delta}(p)=\psi_{i, n}^{\delta}(p), \quad p \in P_{2}\left(\gamma_{i}\right)\right\}$. 
Since $\sum_{\ell \in \bar{I}_{i}^{\delta} \cap \tilde{\Delta}} \breve{\sigma}_{\ell}^{n} \breve{\psi}_{i, \ell}^{\delta}(p)=\psi_{i, n}^{\delta}(p)$ for $p \in P_{2}\left(\gamma_{i}\right)$ has a unique, uniformly bounded solution, $\bar{I}_{i}^{\delta} \cap \tilde{\triangle} \subset \bar{I}_{i}^{\delta}(\tilde{\triangle})$, and the transformation from $\left\{\psi_{i, \ell}^{\delta}: \ell \in \bar{I}_{i}^{\delta}(\tilde{\triangle})\right\}$ to $\left\{\breve{\psi}_{i, \ell}^{\delta}: \ell \in \bar{I}_{i}^{\delta}(\tilde{\triangle})\right\}$ is uniformly boundedly invertible, we infer the above constrained minimization problem has a unique solution with $\left|\sigma_{\ell}^{n}\right| \lesssim 1$. We remove $n$ from $\bar{I}_{i}^{\delta}$ and thus $\bar{m}_{i, n}^{\delta}$ from the collection $\left\{\bar{m}_{i, \ell}^{\delta}: \ell \in \bar{I}_{i}^{\delta}\right\}$, and redefine

$$
\bar{m}_{i, \ell}^{\delta} \leftarrow \bar{m}_{i, \ell}^{\delta}+\sigma_{\ell}^{n} \bar{m}_{i, n}^{\delta}, \quad \ell \in \bar{I}_{i}^{\delta}(\tilde{\triangle}) .
$$

After removal of all $n \in \bar{I}_{i}^{\delta} \backslash I_{i}^{\delta}$, the obtained collection $\left\{m_{i, \ell}^{\delta}: \ell \in I_{i}^{\delta}\right\}$ of dual Lagrange multipliers spans a space $M_{i}^{\delta}$ that has the required local approximation properties.

\section{Numerical Results}

In this section, we present some numerical results illustrating the performance of the quadratic tetrahedral and quadratic serendipity hexahedral mortar finite elements from $\S 3.2 .1$ and $\S 3.2 .2$. For the primal variable, we give the discretization errors in the $\|\cdot\|_{1}:=$ $\left[\sum_{k=1}^{K}\|\cdot\|_{H^{1}\left(\Omega_{k}\right)^{\frac{1}{2}}}^{2}\right.$-norm, as also in the $\|\cdot\|_{L^{2}(\Omega)}$-norm. Since we haven't implemented adaptive refinements yet, in order to benefit from higher order elements, in the examples in which we studied convergence rates, we have prescribed piecewise smooth solutions. Using exclusively quasi-uniform partitions, with $h$ denoting the largest diameter of any underlying element, Corollary 2.4 shows that the $\|\cdot\|_{1}$-norm of the error is of order $h^{2}$. Using duality arguments, one can prove, see e.g. [9], that the error in $L^{2}(\Omega)$-norm is of order $h^{3}$. Since the $\|\cdot\|_{\left(H_{00}^{\frac{1}{2}}\left(\gamma_{i}\right)\right)^{\prime}}$-norms are not so easy to compute, we measure the discretization errors in the flux across the interfaces in a meshdependent Lagrange multiplier norm, that for tetrahedral elements is defined by

$$
\|\mu\|_{\delta}^{2}:=\sum_{i=1}^{N} \sum_{\triangle \in \triangle_{i}^{\delta}} \operatorname{diam}(\triangle)\left\|\left.\mu\right|_{\triangle}\right\|_{L^{2}(\triangle)}^{2},
$$

and that has an analogous definition for the hexahedral elements with $\triangle_{i}^{\delta}, \triangle$ replaced by $\square_{i}^{\delta}, \square$. Theoretically, the asymptotic rates of errors in the weighted Lagrange multiplier norm are of order $h^{2}$ for quadratic mortar finite elements, see [19]. However, under the assumption that the errors in the primal variable are equally distributed, which is the 
case here because of the smooth solution, the asymptotic rates can be shown to be of order $h^{5 / 2}$, see [29].

In our examples, we combine tetrahedral and hexahedral elements on the different subdomains. We point out that different Lagrange multipliers are used depending on the hexahedral or tetrahedral partition on the slave side of the interface. Starting with some partition into tetrahedra or hexahedra on each subdomain, we create sequences of partitions by uniform dyadic refinements. We use $I=\mathbb{N}_{0}$ as index set for $\delta$, where then $\delta$ refers to the level of refinement, that is, the number of uniform dyadic refinement steps that has been applied.

For solving the arising linear systems, we have used the multigrid method applied to a reformulation of (1.2) as a positive definite system on the product space $X^{\delta}$ as introduced in [30]. This multigrid method has a level independent convergence rate and is of optimal computational complexity, see [30,28]. Our implementation is based on the finite element toolbox ug, [1].

In all our examples, we observe convergence rates for $\left\|u-u^{\delta}\right\|_{L^{2}(\Omega)}$, $\left\|u-u^{\delta}\right\|_{1}$, and $\left\|\lambda-\lambda^{\delta}\right\|_{\delta}$ that approach the values 8,4 , and $2^{\frac{5}{2}} \approx$ 5.66 , respectively, that, when having smooth solutions, are expected for dyadic refinements and quadratic/linear approximation for the primal/Lagrange multiplier variables.

In our first example, we consider $-\Delta u=f$ in $\Omega$, where $\Omega$ is composed of five cubes $\Omega_{1}:=(0,1)^{3}, \Omega_{2}:=(1,2) \times(0,1)^{2}, \Omega_{3}:=$ $(0,1)^{2} \times(1,2), \Omega_{4}:=(-1,0) \times(0,1)^{2}$ and $\Omega_{5}:=(0,1)^{2} \times(-1,0)$. Here, subdomain $\Omega_{1}$, which has an initial partition into 27 hexahedra, is the slave subdomain and the others are master subdomains. In each of the master subdomains, the initial partition consists of 6 tetrahedra. The right hand side $f$ and the Dirichlet boundary conditions are chosen such that the exact solution is given by

$$
u(x, y, z)=e^{-0.25\left(x^{2}+y^{2}+z^{2}\right)}(\cos (5 x+z)+3 \sin (4 y+z)) .
$$

In Figure 4.1, the decomposition of the domain, the initial finite element partitions, and the isolines of the solution at the interface $z=1$ are shown. The discretization errors are given in Table 4.1.

In our second example, we consider a domain and problem from [19]. Here, $\Omega:=(0,2) \times(0,1) \times(0,2)$ is decomposed into four subdomains $\Omega_{1}:=(0,1)^{3}, \Omega_{2}:=(0,1)^{2} \times(1,2), \Omega_{3}:=(1,2) \times(0,1)^{2}$ and $\Omega_{4}:=(1,2) \times(0,1) \times(1,2)$, with $\Omega_{2}$ and $\Omega_{3}$ being slave subdomains, and $\Omega_{1}$ and $\Omega_{4}$ being master subdomains. On both $\Omega_{2}$ and $\Omega_{3}$ the initial partition consists of 27 hexahedra, and on both $\Omega_{1}$ and $\Omega_{4}$ it consists of 6 hexahedra. The problem for this example is given by a 

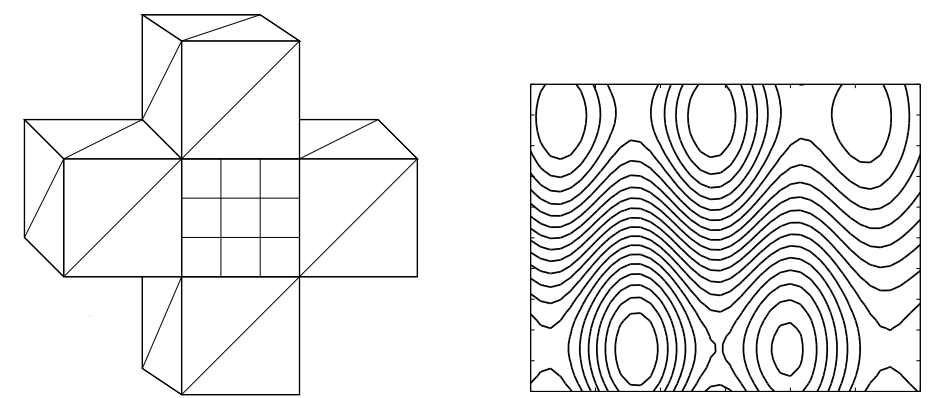

Fig. 4.1: Decomposition of the domain and initial partitions (left), isolines of the solution at the plane $z=1$ (right) for Example 1.

Table 4.1. Discretization errors for Example 1.

\begin{tabular}{|c|c|c|c|c|c|c|c|}
\hline$\delta$ & $\#$ elem. & \multicolumn{2}{|c|}{$\left\|u-u^{\delta}\right\|_{L^{2}(\Omega)}$} & \multicolumn{2}{|c|}{$\left\|u-u^{\delta}\right\|_{1}$} & \multicolumn{2}{|c|}{$\left\|\lambda-\lambda^{\delta}\right\|_{\delta}$} \\
\hline 0 & 51 & $3.24 \mathrm{e}-1$ & & $5.75 \mathrm{e}-1$ & & $3.20 \mathrm{e}+0$ & \\
\hline 1 & 408 & $5.02 \mathrm{e}-2$ & 6.5 & $1.70 \mathrm{e}-1$ & 3.4 & $8.44 \mathrm{e}-1$ & 3.8 \\
\hline 2 & 3264 & $6.79 \mathrm{e}-3$ & 7.4 & $4.70 \mathrm{e}-2$ & 3.6 & $1.60 \mathrm{e}-1$ & 5.3 \\
\hline 3 & 26112 & $8.52 \mathrm{e}-4$ & 8.0 & $1.19 \mathrm{e}-2$ & 3.9 & $2.94 \mathrm{e}-2$ & 5.4 \\
\hline
\end{tabular}

reaction-diffusion equation

$$
-\operatorname{div}(a \nabla u)+u=f \quad \text { in } \quad \Omega,
$$

where $a=1$ in $\Omega_{1}$ and $\Omega_{4}$, and $a=10$ in $\Omega_{2}$ and $\Omega_{3}$. The right hand side $f$ and the Dirichlet boundary conditions are chosen such that the exact solution is given by

$$
u(x, y, z)=(x-1) y(z-1) e^{-(x-1)^{2}-y^{2}-(z-1)^{2}} \cos (2 x+2 y+2 z) / a .
$$

Note that $u$ has a jump in the normal derivative across the interface, whereas the flux is continuous. In Figure 4.2, the decomposition of the domain together with the initial finite element partitions, the isolines of the solution on the plane $y=\frac{1}{2}$, and the flux of the exact solution at the interface $x=1$ are shown. The discretization errors are given in Tables 4.2.

Our third example is given by $-\Delta u=f$ in $\Omega$, where the nonconvex $\Omega$ is composed of eight cubes $\Omega_{1}:=(0,1)^{3}, \Omega_{2}:=(1,2) \times$ $(0,1)^{2}, \Omega_{3}:=(2,3) \times(0,1)^{2}, \Omega_{4}:=(0,1)^{2} \times(1,3), \Omega_{5}:=(2,3) \times$ $(0,1) \times(1,3), \Omega_{6}:=(0,1)^{2} \times(3,4), \Omega_{7}:=(1,2) \times(0,1) \times(3,4)$ and $\Omega_{8}:=(2,3) \times(0,1) \times(3,4)$. Here, subdomains $\Omega_{1}, \Omega_{3}, \Omega_{6}$ and $\Omega_{8}$ are taken as slave subdomains and the others are master subdomains. The initial partitions consist of tetrahedra for $\Omega_{1}$ and $\Omega_{3}$, and of 

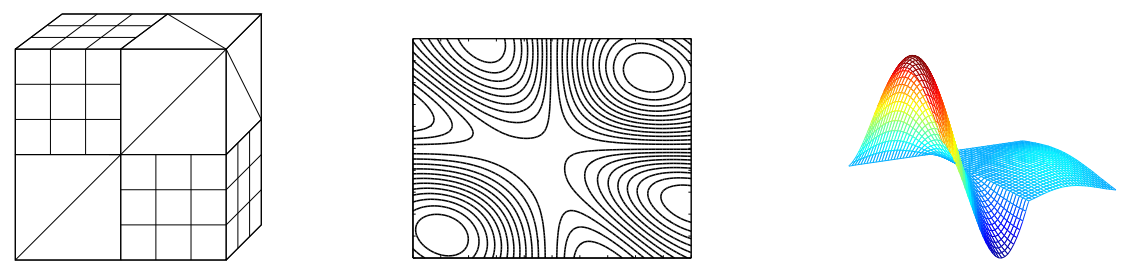

Fig. 4.2: Decomposition of the domain and initial partitions (left), isolines of the solution at the plane $y=\frac{1}{2}$ (middle) and flux of the exact solution at the interface $x=1$ (right) for Example 2 .

Table 4.2. Discretization errors for Example 2.

\begin{tabular}{|c|c|c|c|c|c|c|c|}
\hline$\delta$ & \# elem. & \multicolumn{2}{|c|}{$\left\|u-u^{\delta}\right\|_{L^{2}(\Omega)}$} & \multicolumn{2}{|c|}{$\left\|u-u^{\delta}\right\|_{1}$} & \multicolumn{2}{|c|}{$\left\|\lambda-\lambda^{\delta}\right\|_{\delta}$} \\
\hline 0 & 66 & $7.94 \mathrm{e}-2$ & & $1.57 \mathrm{e}-1$ & & $5.19 \mathrm{e}-2$ & \\
\hline 1 & 528 & $1.69 \mathrm{e}-2$ & 4.7 & $4.99 \mathrm{e}-2$ & 3.1 & $4.11 \mathrm{e}-3$ & 13 \\
\hline 2 & 4224 & $2.35 \mathrm{e}-3$ & 7.2 & $1.48 \mathrm{e}-2$ & 3.4 & $6.26 \mathrm{e}-4$ & 6.5 \\
\hline 3 & 33792 & $2.73 \mathrm{e}-4$ & 8.6 & $3.87 \mathrm{e}-3$ & 3.8 & $1.04 \mathrm{e}-4$ & 6.0 \\
\hline
\end{tabular}

hexahedra for the other subdomains, and they are illustrated in Figure 4.3. The right hand side $f$ and the Dirichlet boundary conditions are chosen such that the exact solution is given by

$$
u(x, y, z)=e^{-\frac{1}{4}(x+y+z)}(\sin (5 x y)+\cos (5 y z))\left(x+y^{2}+z^{2}\right) .
$$

The isolines of this solution at the interface $z=1$ can also be found in Figure 4.3. Finally, the discretization errors are given in Table 4.3.
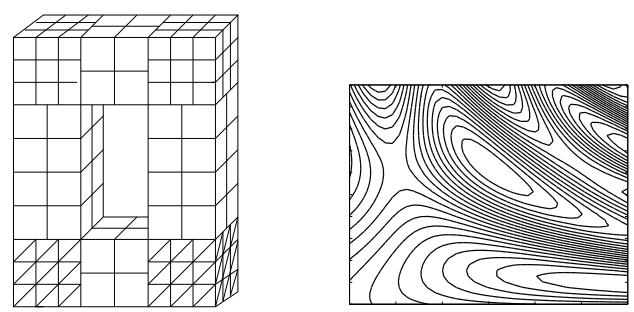

Fig. 4.3: Decomposition of the domain and initial partitions (left), isolines of the solution at the plane $z=1$ (right) for Example 3 .

In our last example, we consider an example in linear elasticity. We point out that the theory can easily be extended to this case with standard arguments. For this example, we take the domain and the problem from [17]. Here, the computational domain $\Omega$, which is a beam, is decomposed into three subdomains $\Omega_{1}, \Omega_{2}$ and $\Omega_{3}$ with 
Table 4.3. Discretization errors for Example 3.

\begin{tabular}{|c|c|c|c|c|c|c|c|}
\hline$\delta$ & \# elem. & $\left\|u-u^{\delta}\right\|_{L^{2}(\Omega)}$ & \multicolumn{2}{|c|}{$\left\|u-u^{\delta}\right\|_{1}$} & \multicolumn{2}{|c|}{$\left\|\lambda-\lambda^{\delta}\right\|_{\delta}$} \\
\hline 0 & 426 & $6.94 \mathrm{e}-1$ & & $9.13 \mathrm{e}-1$ & & $1.07 \mathrm{e}+1$ & \\
\hline 1 & 3408 & $1.43 \mathrm{e}-1$ & 4.9 & $4.18 \mathrm{e}-1$ & 2.2 & $5.79 \mathrm{e}+0$ & 1.8 \\
\hline 2 & 27264 & $1.98 \mathrm{e}-2$ & 7.2 & $1.10 \mathrm{e}-1$ & 3.8 & $1.34 \mathrm{e}+0$ & 4.3 \\
\hline 3 & 218112 & $2.63 \mathrm{e}-3$ & 7.5 & $2.85 \mathrm{e}-2$ & 3.9 & $1.71 \mathrm{e}-1$ & 7.8 \\
\hline
\end{tabular}

$\Omega_{1}:=(0,50) \times(0,10) \times(0,2), \Omega_{2}:=(0,50) \times(3,7) \times(2,11)$ and $\Omega_{3}:=(0,50) \times(0,10) \times(11,13)$. The decomposition of the domain, is given in the left picture of Figure 4 with an initial partition. Here, $\Omega_{2}$ is the slave subdomain, so that, although the decomposition is geometrically nonconforming, condition (A.1) is satisfied. Our linear elasticity problem is

$$
-\nabla \cdot \sigma(\mathbf{u})=0 \quad \text { in } \quad \Omega
$$

with $\mathbf{u}=0$ on $\Gamma_{D}$ and $\sigma(\mathbf{u}) \mathbf{n}=\mathbf{f}$ on $\Gamma_{N}$, where $\sigma(\mathbf{u})$ is a second order tensor defined as

$$
\sigma(\mathbf{u})=2 \mu \varepsilon(\mathbf{u})+\lambda \operatorname{trace} \varepsilon(\mathbf{u}) I, \quad \varepsilon(\mathbf{u})=\frac{1}{2}\left(\nabla \mathbf{u}+[\nabla \mathbf{u}]^{T}\right)
$$

with $\mu=8.2, \lambda=10\left(\mathrm{~kg} / \mathrm{cm}^{3}\right)$, and $I$ is the identity matrix of size $3 \times 3$. Here, $\Gamma_{D}$ is the part of the boundary of $\Omega$ with $x=0$ and $x=50$ so that the left and the right sides of each subdomain are fixed, and $\Gamma_{N}:=\partial \Omega \backslash \Gamma_{D}$. The function $\mathbf{f}=\left(f_{1}, f_{2}, f_{3}\right)$ on $\Gamma_{N}$ is given as $f_{1}=f_{2}=0$, and

$$
f_{3}= \begin{cases}-0.35 & \text { if } 22 \leq x \leq 28 \text { and } z=13 \\ 0 & \text { otherwise }\end{cases}
$$

so that a constant vertical force is applied from the top boundary $(z=13)$ of $\Omega$. We have computed the solution with quadratic finite elements. The resulting deformation of the beam is given in the right picture of Figure 4.

Acknowledgements The authors thank the anonymous referees for their careful reading of an earlier version of this work, pointing out some mistakes to us.

\section{References}

1. P. Bastian, K. Birken, K. Johannsen, S. Lang, N. Neuß, H. Rentz-Reichert, and C. Wieners. UG - a flexible software toolbox for solving partial differential equations. Computing and Visualization in Science, 1:27-40, 1997. 

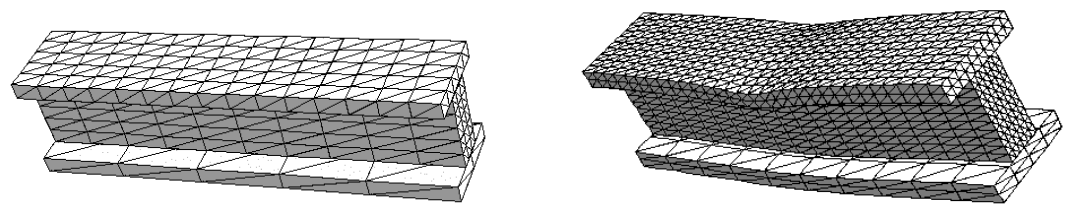

Fig. 4.4: Decomposition of the domain with initial partition (left) and the distorted mesh after refining once (right)

2. F. Ben Belgacem. The mortar finite element method with Lagrange multipliers. Numer. Math., 84:173-197, 1999.

3. F. Ben Belgacem and Y. Maday. The mortar element method for three dimensional finite elements. $M^{2} A N, 31: 289-302,1997$.

4. F. Ben Belgacem, P. Seshaiyer, and M. Suri. Optimal convergence rates of $h p$ mortar finite element methods for second-order elliptic problems. M2AN Math. Model. Numer. Anal., 34(3):591-608, 2000.

5. C. Bernardi, N. Debit, and Y. Maday. Coupling finite element and spectral methods: First results. Math. Comp., 54:21-39, 1990.

6. C. Bernardi, Y. Maday, and A.T. Patera. Domain decomposition by the mortar element method. In H. Kaper et al., editor, Asymptotic and numerical methods for partial differential equations with critical parameters, pages 269286. Reidel, Dordrecht, 1993.

7. C. Bernardi, Y. Maday, and A.T. Patera. A new nonconforming approach to domain decomposition: the mortar element method. In H. Brezzi et al., editor, Nonlinear partial differential equations and their applications, pages 13-51. Paris, 1994.

8. D. Braess and W. Dahmen. Stability estimates of the mortar finite element method for 3-dimensional problems. East-West J. Numer. Math., 6:249-264, 1998.

9. D. Braess, W. Dahmen, and C. Wieners. A multigrid algorithm for the mortar finite element method. SIAM J. Numer. Anal., 37:48-69, 1999.

10. F. Brezzi and M. Fortin. Mixed and hybrid finite element methods. SpringerVerlag, New York, 1991.

11. P. Clément. Approximation by finite element functions using local regularization. RAIRO Anal. Numér., 9:77-84, 1975.

12. W. Dahmen, B. Faermann, I.G. Graham, W. Hackbusch, and S.A. Sauter. Inverse inequalities on non-quasiuniform meshes and application to the mortar element method. Math. Comp., 73:1107-1138, 2004.

13. R. DeVore. Nonlinear approximation. Acta Numer., 7:51-150, 1998.

14. J. Gopalakrishnan and J. Pasciak. Multigrid for the mortar finite element method. SIAM J. Numer. Anal., 37:1029-1052, 2000.

15. P. Grisvard. Elliptic problems in nonsmooth domains, volume 24 of Monographs and Studies in Mathematics. Pitman (Advanced Publishing Program), Boston, MA, 1985.

16. J. Huang and J. Zou. A mortar element method for elliptic problems with discontinuous coefficients. IMA J. Numer. Anal., 22(4):549-576, 2002.

17. C. Kim, R.D. Lazarov, J.E. Pasciak, and P.S. Vassilevski. Multiplier spaces for the mortar finite element method in three dimensions. SIAM J. Numer. Anal., 39:519-538, 2001. 
18. B.P. Lamichhane and B.I. Wohlmuth. Higher order dual Lagrange multiplier spaces for mortar finite element discretizations. CALCOLO, 39:219-237, 2002.

19. B.P. Lamichhane and B.I. Wohlmuth. A quasi-dual Lagrange multiplier space for serendipity mortar finite elements in 3D. $M^{2} A N, 38: 73-92,2004$.

20. J.-L. Lions and E. Magenes. Non-homogeneous boundary value problems and applications. Vol. I. Springer-Verlag, New York, 1972. Translated from the French by P. Kenneth, Die Grundlehren der mathematischen Wissenschaften, Band 181.

21. P. Oswald. Multilevel finite element approximation: Theory and applications. B.G. Teubner, Stuttgart, 1994.

22. P. Oswald and B.I. Wohlmuth. On polynomial reproduction of dual FE bases. In N. Debit, M. Garbey, R. Hoppe, J. Pèriaux, D. Keyes, and Y. Kuznetsov, editors, Thirteenth International Conference on Domain Decomposition Methods, pages 85-96, 2001.

23. L. R. Scott and S. Zhang. Finite element interpolation of nonsmooth functions satisfying boundary conditions. Math. Comp., 54(190):483-493, 1990.

24. P. Seshaiyer and M. Suri. Uniform $h p$ convergence results for the mortar finite element method. Math. Comp., 69(230):521-546, 2000.

25. H. Triebel. Interpolation theory, function spaces, differential operators, volume 18 of North-Holland Mathematical Library. North-Holland Publishing Co., Amsterdam, 1978.

26. C. Wieners and B.I. Wohlmuth. Duality estimates and multigrid analysis for saddle point problems arising from mortar discretizations. SISC, 24:21632184, 2003.

27. B.I. Wohlmuth. A mortar finite element method using dual spaces for the Lagrange multiplier. SIAM J. Numer. Anal., 38:989-1012, 2000.

28. B.I. Wohlmuth. Discretization Methods and Iterative Solvers Based on Domain Decomposition, volume 17 of LNCS. Springer Heidelberg, 2001.

29. B.I. Wohlmuth. A comparison of dual Lagrange multiplier spaces for mortar finite element discretizations. $M^{2} A N, 36: 995-1012,2002$.

30. B.I. Wohlmuth and R.H. Krause. Multigrid methods based on the unconstrained product space arising from mortar finite element discretizations. SIAM J. Numer. Anal., 39:192-213, 2001. 